\title{
REGULARITY AND MULTIGRID ANALYSIS FOR LAPLACE-TYPE AXISYMMETRIC EQUATIONS
}

By

Hengguang Li

\section{IMA Preprint Series \#2417}

(February 2013)

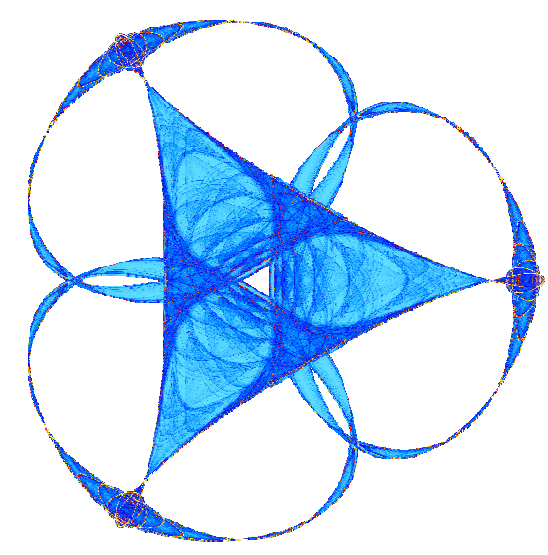

INSTITUTE FOR MATHEMATICS AND ITS APPLICATIONS

UNIVERSITY OF MINNESOTA 400 Lind Hall

207 Church Street S.E.

Minneapolis, Minnesota 55455-0436

Phone: 612-624-6066 Fax: 612-626-7370

URL: http://www.ima.umn.edu 


\title{
REGULARITY AND MULTIGRID ANALYSIS FOR LAPLACE-TYPE AXISYMMETRIC EQUATIONS
}

\author{
HENGGUANG LI
}

\begin{abstract}
Consider axisymmetric equations associated with Laplace-type operators. We establish full regularity estimates in high-order Kondrat'vetype spaces for possible singular solutions due to the non-smoothness of the domain and to the singular coefficients in the operator. Then, we show suitable graded meshes can be used in high-order finite element methods to achieve the optimal convergence rate, even when the solution is singular. Using these results, we further propose multigrid V-cycle algorithms solving the system from linear finite element discretizations on optimal graded meshes. We prove the multigrid algorithm is a contraction, with the contraction number independent of the mesh level. Numerical tests are provided to verify the theorem.
\end{abstract}

\section{INTRODUCTION}

Let $\Omega \subset \mathbb{R}_{+}^{2}:=\{(r, z), r>0\}$ be a bounded polygonal domain in the $r z$-plane and the integer $\beta=0$ or 1 . Let $\Gamma_{0}$ be the interior part of $\partial \Omega \cap\{r=0\}$ and $\Gamma_{1}:=\partial \Omega \backslash \Gamma_{0}$. See Figure 1 for example. We consider equations with parameter $\beta$,

$$
\mathcal{L}_{\beta} u_{\beta}:=-\left(\partial_{r}^{2}+r^{-1} \partial_{r}+\partial_{z}^{2}-\beta r^{-2 \beta}\right) u_{\beta}=f_{\beta} \quad \text { in } \quad \Omega,
$$

with the $\beta$-dependent boundary conditions

$$
\begin{gathered}
u_{0}=0 \quad \text { on } \quad \Gamma_{1}, \quad \partial_{r} u_{0}=0 \text { on } \Gamma_{0} ; \\
u_{1}=0 \quad \text { on } \partial \Omega .
\end{gathered}
$$

Equation (1) plays an important role in the study of various equations in 3D axisymmetric domains. For instance, denote by $\mathbb{R}^{3} \ni \tilde{\Omega}:=\left(\Omega \cup \Gamma_{0}\right) \times[0,2 \pi)$ the domain obtained by the rotation of $\Omega$ (meridian domain) about the $z$-axis (Figure $1)$. Recall the Cartesian coordinates $(x, y, z)$ and the cylindrical coordinates $(r, \theta, z)$ on $\tilde{\Omega}$. For any $\tilde{v} \in L^{2}(\tilde{\Omega})$, its Fourier coefficients with respect to $\theta$ are defined by

$$
v_{k}(r, z)=\mathcal{F}_{k}(\tilde{v}):=\frac{1}{\sqrt{2 \pi}} \int_{-\pi}^{\pi} \tilde{v}(r, \theta, z) e^{-i k \theta} d \theta, \quad k \in \mathbb{Z} .
$$

Let $\tilde{w}$ be the solution of the 3D Poisson equation

$$
-\Delta \tilde{w}=\tilde{f} \quad \text { in } \quad \tilde{\Omega}, \quad \tilde{w}=0 \quad \text { on } \partial \tilde{\Omega} .
$$

Then, the first three Fourier coefficients $w_{k},|k| \leq 1$, of $\tilde{w}$ are given by equation (1) as follows: $w_{0}=u_{0}$, given $f_{0}=\mathcal{F}_{0}(\tilde{f})(\beta=0) ; w_{1}=u_{1}$, given $f_{1}=\mathcal{F}_{1}(\tilde{f})$ $(\beta=1)$; and $w_{-1}=u_{1}$, given $f_{1}=\mathcal{F}_{-1}(\tilde{f})(\beta=1)$. In particular, when the 3D data are axisymmetric, $u_{0}$ is the meridian trace of $\tilde{w}$ and $\mathcal{L}_{1} u_{1}=f_{1}$ is regarded as

Date: February 12, 2013.

H. Li was supported in part by the NSF grant DMS-1158839. 

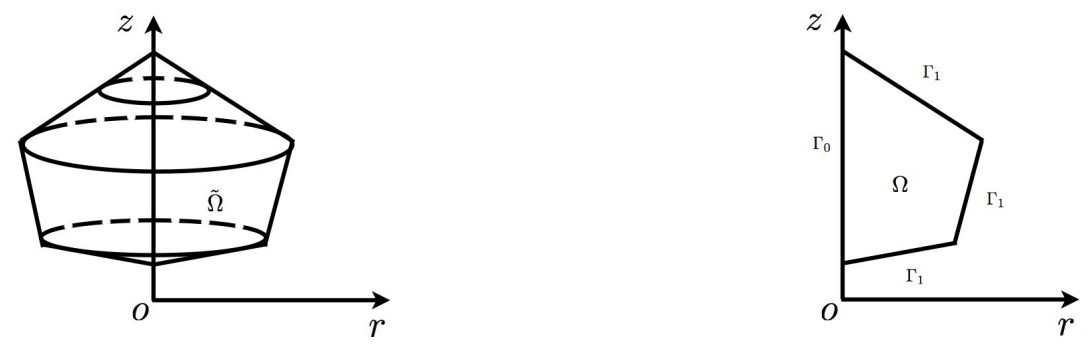

Figure 1. The $3 \mathrm{D}$ axisymmetric domain $\tilde{\Omega}$ (left); the meridian polygonal domain $\Omega$ (right).

the azimuthal Stokes equation $[21,22]$. We also mention that $\mathcal{L}_{1}$ coincides with the principal part of the azimuthal Maxwell operator $[6,15]$.

This dimension reduction from 3D to 2D has the potential for substantial computational savings in numerical approximations of equations on 3D axisymmetric domains. However, the coordinate transformation introduces new differential operators with singular coefficients as in (1) and new function spaces with singular or degenerate weights. The development of robust numerical methods for these equations calls for rigorous numerical analysis, which has recently drawn much attention from the scientific community. For example, [6] provided a comprehensive introduction on spectral methods for various axisymmetric equations. Under the assumption that the solution has sufficient regularity, finite element analysis for axisymmetric problems was discussed in $[1,5,12,21,26]$. In the case that the solution possesses singularities, the singular expansion of the solution for equation (1) was studied in $[17,27]$. As the most relevant results, we mention the papers [23] and [15], both for equation (1) with $\beta=0$. In [23], a second-order regularity estimate was established in a class of weighted (Kondrat'ev-type) spaces for singular solutions. Consequently, the author proposed new linear finite element methods approximating singular solutions in the optimal rate. In [15], the multigrid V-cycle algorithm was studied for finite element spaces augmented with non-polynomial functions. The result was derived only for solutions with required regularity.

This paper contains our systematic study for equation (1) on its regularity, finite element approximation, and multigrid analysis. Motivated by [18, 24, 22], we first introduce high-order Kondrat'ev-type weighted spaces (Definition 2.2) to handle possible singular solutions from the non-smoothness of the domain and from the singular coefficients. Using appropriate isomorphic mappings in weighted spaces, we prove the full-regularity estimates (Theorem 3.5) for singular solutions of equation (1). Based on this regularity result and properties of interpolation operators in weighted spaces, we give an explicit construction of nested graded meshes, such that the associated high-order finite element methods achieve the optimal convergence rate for singular solutions (Theorem 3.16). Then, we analyze the system from the linear finite element discretization on optimal graded meshes. With the growth rate of the condition numbers for nested subspaces (Lemma 4.5), we provide smoothing properties of the Richardson smoother and approximation properties of the numerical solution in various weighted spaces. This leads to our convergence result (Theorem 4.12) on the multigrid V-cycle algorithm for singular solutions of equation (1). 
For equation (1), our high-order regularity result and optimal high-order finite element methods approximating singular solutions are new. These results generalize the results in [23] (low-order regularity and linear finite element results only for the case $\beta=0)$. The uniform convergence of the proposed multigrid method on graded meshes is also new for the axisymmetric equation (1), which recovers the classical multigrid result for elliptic equations with full-regularity solutions [7, 11]. We mention that high-order Fourier coefficients $(|k| \geq 2)$ of $\tilde{w}$ in $(5)$ are determined by equations similar to equation (1) with $\beta=1[6]$. Therefore, our analysis shall provide building blocks for approximating general $2 \mathrm{D}$ Fourier coefficients of the $3 \mathrm{D}$ problem (5). The multigrid method studied here shall be a crucial ingredient in developing efficient multigrid solves for more complex axisymmetric problems.

The rest of the paper is organized as follows. In Section 2, we introduce weighted Sobolev spaces for our problem. We also summarize estimates for functions in these spaces. In Section 3, we establish high-order regularity results in Kondrat'evtype weighted spaces for the possible singular solution of equation (1). Then, we give high-order finite element methods approximating the singular solutions in the optimal rate. In Section 4, we first describe the multigrid V-cycle algorithm for equation (1). Then, we show the proposed multigrid algorithm converges uniformly, independent of the mesh level, even when the solution is singuar. In Section 5, we include multiple numerical tests for the multigrid method to verify the theory.

Acknowledgements. The author would like to thank Jay Gopalakrishnan for discussions and suggestions on this research.

\section{Preliminaries And notation}

We introduce appropriate weighted spaces to study equation (1). Useful estimates regarding these spaces will be collected in the second part of this section.

2.1. Function spaces. We first recall a class of weighted Sobolev spaces from [6].

Definition 2.1. (Type I weighted spaces). For an integer $m \geq 0$, define

$$
L_{1}^{2}(\Omega):=\left\{v, \int_{\Omega} v^{2} r d r d z<\infty\right\}, \quad H_{1}^{m}(\Omega):=\left\{v, \partial_{c}^{\alpha} v \in L_{1}^{2}(\Omega),|\alpha| \leq m\right\},
$$

where the muti-index $\alpha=\left(\alpha_{1}, \alpha_{2}\right)$ is a pair of nonnegative integers, $|\alpha|=\alpha_{1}+\alpha_{2}$, and $\partial_{c}^{\alpha}=\partial_{r}^{\alpha_{1}} \partial_{z}^{\alpha_{2}}$. The norms and the semi-norms for any $v \in H_{1}^{m}(\Omega)$ are

$$
\|v\|_{H_{1}^{m}(\Omega)}^{2}:=\sum_{|\alpha| \leq m} \int_{\Omega}\left(\partial_{c}^{\alpha} v\right)^{2} r d r d z, \quad|v|_{H_{1}^{m}(\Omega)}^{2}:=\sum_{|\alpha|=m} \int_{\Omega}\left(\partial_{c}^{\alpha} v\right)^{2} r d r d z .
$$

Furthermore, we define two spaces $H_{+}^{m}(\Omega)$ and $H_{-}^{m}(\Omega)$.

For $H_{+}^{m}(\Omega)$, if $m$ is not even,

$$
\begin{gathered}
H_{+}^{m}(\Omega):=\left\{v \in H_{1}^{m}(\Omega),\left.\partial_{r}^{2 i-1} v\right|_{\{r=0\}}=0,1 \leq i<\frac{m}{2}\right\}, \\
\|v\|_{H_{+}^{m}(\Omega)}=\|v\|_{H_{1}^{m}(\Omega)}
\end{gathered}
$$

if $m$ is even, besides the condition in (6), we require $\int_{\Omega}\left(\partial_{r}^{m-1} v\right)^{2} r^{-1} d r d z<\infty$ for any $v \in H_{+}^{m}(\Omega)$, and the corresponding norm is

$$
\|v\|_{H_{+}^{m}(\Omega)}=\left(\|v\|_{H_{1}^{m}(\Omega)}^{2}+\int_{\Omega}\left(\partial_{r}^{m-1} v\right)^{2} r^{-1} d r d z\right)^{1 / 2} .
$$


For $H_{-}^{m}(\Omega)$, if $m$ is not odd,

$$
\begin{gathered}
H_{-}^{m}(\Omega):=\left\{v \in H_{1}^{m}(\Omega),\left.\partial_{r}^{2 i} v\right|_{\{r=0\}}=0,0 \leq i<\frac{m-1}{2}\right\} \\
\|v\|_{H_{-}^{m}(\Omega)}=\|v\|_{H_{1}^{m}(\Omega)}
\end{gathered}
$$

if $m$ is odd, besides the condition in (7), we require $\int_{\Omega}\left(\partial_{r}^{m-1} v\right)^{2} r^{-1} d r d z<\infty$, for any $v \in H_{-}^{m}(\Omega)$, and the corresponding norm is

$$
\|v\|_{H_{-}^{m}(\Omega)}=\left(\|v\|_{H_{1}^{m}(\Omega)}^{2}+\int_{\Omega}\left(\partial_{r}^{m-1} v\right)^{2} r^{-1} d r d z\right)^{1 / 2} .
$$

Thus, we denote different subspaces:

$$
H_{+, 0}^{1}(\Omega):=H_{+}^{1}(\Omega) \cap\left\{\left.v\right|_{\Gamma_{1}}=0\right\}, \quad H_{-, 0}^{1}(\Omega):=H_{-}^{1}(\Omega) \cap\left\{\left.v\right|_{\partial \Omega}=0\right\} .
$$

We now introduce another type of weighted spaces for our analysis on singular solutions of equation (1).

Definition 2.2. (Type II weighted spaces). Let $Q_{i}$ be the $i$ th vertex of $\Omega$ and define the vertex set $\mathcal{Q}:=\left\{Q_{i}\right\}_{i=1}^{I}$. Denote by $2 L$ the smallest distance from a vertex to any disjoint edge of $\partial \Omega$. Let $B\left(x, r_{0}\right)$ be the ball centered at $x$ with radius $r_{0}$. Let $\vartheta \in \mathcal{C}^{\infty}(\bar{\Omega} \backslash \mathcal{Q})$ be a function, such that $\vartheta=\left|x-Q_{i}\right|$ in $\mathcal{V}_{i}:=\Omega \cap B\left(Q_{i}, L / 2\right)$ and $\vartheta \geq L / 2$ in $\Omega \backslash \cup_{i=1}^{I} \mathcal{V}_{i}$. Note that $\mathcal{V}_{i}$ and $\mathcal{V}_{j}$ are disjoint if $i \neq j$. Thus, we define for $\mu \in \mathbb{R}$ and for any open set $G \subset \Omega$,

$$
\mathcal{K}_{\mu, 1}^{m}(G):=\left\{v, \vartheta^{-\mu+|\alpha|} \partial_{c}^{\alpha} v \in L_{1}^{2}(G),|\alpha| \leq m\right\} .
$$

with the semi-norm and norm

$$
|v|_{\mathcal{K}_{\mu, 1}^{m}(G)}^{2}:=\sum_{|\alpha|=m}\left\|\vartheta^{m-\mu} \partial_{c}^{\alpha} v\right\|_{L_{1}^{2}(G)}^{2}, \quad\|v\|_{\mathcal{K}_{\mu, 1}^{m}(G)}^{2}:=\sum_{l=0}^{m}|v|_{\mathcal{K}_{\mu, 1}^{l}(G)}^{2} .
$$

In addition, for $0 \leq l \leq m$, we define the subspaces of $\mathcal{K}_{\mu, 1}^{m}(\Omega)$ :

(8) $\mathcal{K}_{\mu,+}^{m}(\Omega):=\left\{v \in \mathcal{K}_{\mu, 1}^{m}(\Omega), \int_{\Omega}\left(\partial_{r}^{2 i-1}\left(\vartheta^{-\mu+l} v\right)\right)^{2} r^{-1} d r d z<\infty, 1 \leq i \leq \frac{l}{2}\right\}$,

(9) $\mathcal{K}_{\mu,-}^{m}(\Omega):=\left\{v \in \mathcal{K}_{\mu, 1}^{m}(\Omega), \int_{\Omega}\left(\partial_{r}^{2 i}\left(\vartheta^{-\mu+l} v\right)\right)^{2} r^{-1} d r d z<\infty, 0 \leq i \leq \frac{l-1}{2}\right\}$.

The corresponding norms are

$$
\begin{gathered}
\|v\|_{\mathcal{K}_{\mu,+}^{m}(\Omega)}=\left(\|v\|_{\mathcal{K}_{\mu, 1}^{m}(\Omega)}^{2}+\sum_{l \leq m} \sum_{1 \leq i \leq \frac{l}{2}} \int_{\Omega}\left(\partial_{r}^{2 i-1}\left(\vartheta^{-\mu+l} v\right)\right)^{2} r^{-1} d r d z\right)^{1 / 2} \\
\|v\|_{\mathcal{K}_{\mu,-}^{m}(\Omega)}=\left(\|v\|_{\mathcal{K}_{\mu, 1}^{m}(\Omega)}^{2}+\sum_{l \leq m} \sum_{0 \leq i \leq \frac{l-1}{2}} \int_{\Omega}\left(\partial_{r}^{2 i}\left(\vartheta^{-\mu+l} v\right)\right)^{2} r^{-1} d r d z\right)^{1 / 2} .
\end{gathered}
$$

Recall the Fourier coefficients $v_{k}$ of $\tilde{v}$ in (4). We then have the following isomorphism (Theorem II.3.1) [6].

Proposition 2.3. For $m \geq 0, \tilde{v} \rightarrow\left(v_{k}\right)_{k \in \mathbb{Z}}$ defines an isomorphic mapping

$$
H^{m}(\tilde{\Omega}) \rightarrow \Pi_{k \in \mathbb{Z}} H_{(k)}^{m}(\Omega),
$$

where the space $H_{(k)}^{m}(\Omega)$ is defined as follows. For $|k|>m-1$,

$$
\|w\|_{H_{(k)}^{m}(\Omega)}^{2}=\|w\|_{H_{1}^{m}(\Omega)}^{2}+|k|^{2 m}\left\|r^{-m} w\right\|_{L_{1}^{2}(\Omega)}^{2} .
$$




$$
\begin{aligned}
& \text { If }|k| \leq m-1, \text { for } 0 \leq j \leq|k|-1, \\
& \quad H_{(k)}^{m}(\Omega)=H_{+}^{m}(\Omega) \cap\left\{\left.\partial_{r}^{j} w\right|_{\Gamma_{0}}=0\right\} \text { and }\|w\|_{H_{(k)}^{m}(\Omega)}=\|w\|_{H_{+}^{m}(\Omega)} \quad(\text { even } k) \\
& \quad H_{(k)}^{m}(\Omega)=H_{-}^{m}(\Omega) \cap\left\{\left.\partial_{r}^{j} w\right|_{\Gamma_{0}}=0\right\} \text { and }\|w\|_{H_{(k)}^{m}(\Omega)}=\|w\|_{H_{-}^{m}(\Omega)} \quad(\text { odd } k) .
\end{aligned}
$$

Remark 2.4. For $\tilde{v} \in H^{m}(\tilde{\Omega}), H_{(k)}^{m}(\Omega)$ are natural spaces to study the traces of its Fourier coefficients on $\Omega$. We shall show that the Kondrat'ev-type spaces $\mathcal{K}_{\mu, 1}^{m}(\Omega)$ are the appropriate spaces to study the trace when $\tilde{v}$ only has reduced regularity. Note that for $m \geq 0$,

$$
H_{(0)}^{m}(\Omega)=H_{+}^{m}(\Omega), \quad H_{( \pm 1)}^{m}(\Omega)=H_{-}^{m}(\Omega) .
$$

Thus, we shall consider equation (1) in $H_{+}^{m}(\Omega)$ if $\beta=0$ and in $H_{-}^{m}(\Omega)$ if $\beta=$ 1. Inheriting from the $3 \mathrm{D}$ Poisson equation (5), we have the boundary condition $\left.u_{\beta}\right|_{\Gamma_{1}}=0$. The restrictions on $u_{\beta}$ in type I weighted spaces lead to different boundary conditions on $\Gamma_{0}:=\partial \Omega \backslash \Gamma_{1}$ in (2) and (3). Since the Fourier coefficients may be complex even though the original function is real, we shall denote the complex extensions of all the spaces above without change of notation.

The Fourier transform (4) leads to the variational formulation for equation (1) [6]: Find $u_{0} \in H_{+, 0}^{1}(\Omega)$ for any $v_{0} \in H_{+, 0}^{1}(\Omega)\left(\right.$ resp. $u_{1} \in H_{-, 0}^{1}(\Omega)$ for $v_{1} \in H_{-, 0}^{1}(\Omega)$ ), such that

$$
a_{\beta}\left(u_{\beta}, v_{\beta}\right):=\int_{\Omega}\left(\partial_{r} u_{\beta} \partial_{r} v_{\beta}+\partial_{z} u_{\beta} \partial_{z} v_{\beta}+\frac{\beta}{r^{2}} u_{\beta} v_{\beta}\right) r d r d z=\left\langle f_{\beta}, v_{\beta}\right\rangle,
$$

where $f_{0} \in H_{+, 0}^{1}(\Omega)^{\prime}$ (resp. $\left.f_{1} \in H_{-, 0}^{1}(\Omega)^{\prime}\right)$, the dual space of $H_{+, 0}^{1}(\Omega)$ (resp. $H_{-, 0}^{1}(\Omega)$ ) with the pivot space $L_{1}^{2}(\Omega)$. For $f_{\beta} \in L_{1}^{2}(\Omega)$, the right hand side is the $L_{1}^{2}$-inner product. Then, we have the well-posedness result.

Corollary 2.5. The variational formulation (11) defines a unique solution $u_{0} \in$ $H_{+, 0}^{1}(\Omega)$ (resp. $u_{1} \in H_{-, 0}^{1}(\Omega)$ ) and there is a constant $C>0$, independent of $u_{\beta}$ and $f_{\beta}$, such that

$$
\left\|u_{0}\right\|_{H_{+}^{1}(\Omega)} \leq C\left\|f_{0}\right\|_{H_{+, 0}^{1}(\Omega)^{\prime}} \quad \text { and } \quad\left\|u_{1}\right\|_{H_{-}^{1}(\Omega)} \leq C\left\|f_{1}\right\|_{H_{-, 0}^{1}(\Omega)^{\prime}} .
$$

Proof. For $\beta=0$, the well-posedness follows from the isomorphism (10) and the 3D Poincaré inequality; for $\beta=1$, it follows from the fact that $a_{\beta}(\cdot, \cdot)$ is coercive and continuous in $H_{-, 0}^{1}(\Omega)$. The estimates in (12) are immediate consequences of the well-posedness.

2.2. Weighted estimates. We now recall several estimates from [22]. These estimates give relations between various spaces and will be used to formulate our high-order regularity results in the next section.

We distinguish the vertices on and off the $z$-axis as follows. A vertex on the $z$-axis will be denoted by $Q^{z}$; A vertex away from the $z$-axis will be denoted by $Q^{r}$. Recall the neighborhood $\mathcal{V}:=B(Q, L / 2) \cap \Omega$ of the vertex $Q$. For a vertex $Q^{z}$, let $\Gamma_{\mathcal{V}}=\Gamma_{0} \cap \overline{\mathcal{V}}$ and we denote by $\tilde{\mathcal{V}}:=\left(\mathcal{V} \cup \Gamma_{\mathcal{V}}\right) \times[0,2 \pi) \subset \tilde{\Omega}$ the neighborhood of $Q^{z}$ in $\tilde{\Omega}$. For an integer $l \geq 1, \mathcal{V} / l:=B(Q, L /(2 l)) \cap \Omega$ and $\tilde{\mathcal{V}} / l:=\left(\mathcal{V} / l \cup \Gamma_{\mathcal{V} / l}\right) \times[0,2 \pi)$.

Throughout the paper, by $H^{\prime}$, we mean the dual space of $H$. As in Definition 2.1 , we also use the multi-index $\alpha=\left(\alpha_{1}, \alpha_{2}, \alpha_{3}\right)$ for a 3D domain, such that $|\alpha|=$ $\alpha_{1}+\alpha_{2}+\alpha_{3}$ and $\partial^{\alpha}=\partial_{x}^{\alpha_{1}} \partial_{y}^{\alpha_{2}} \partial_{z}^{\alpha_{3}}$. The generic constant $C>0$ in our analysis below may be different at different occurrences. It will depend on the computational 
domain, but not on the functions involved in the estimates or the mesh level in the finite element algorithms.

The following lemma, derived as Lemmas 2.8 and 2.9 in [22], contains useful weighted estimates in usual Sobolev spaces in the $3 \mathrm{D}$ neighborhood $\tilde{\mathcal{V}}$ of a vertex $Q^{z}$. We refer to [22] for the detailed proof.

Lemma 2.6. Let $\tilde{\mathcal{V}} \subset \tilde{\Omega}$ be the neighborhood of a vertex $Q^{z}$ and $\rho$ be the distance to $Q^{z}$. Suppose $\sum_{|\alpha| \leq m}\left\|\rho^{-a+|\alpha|} \partial^{\alpha} v\right\|_{L^{2}(\tilde{\mathcal{V}})}^{2}<\infty$, for $m \geq 0$ and $a \in \mathbb{R}$. Then, for any $0 \leq l \leq m$,

$$
C\left\|\rho^{-a+l} v\right\|_{H^{l}(\tilde{\mathcal{V}})}^{2} \leq \sum_{|\alpha| \leq m}\left\|\rho^{-a+|\alpha|} \partial^{\alpha} v\right\|_{L^{2}(\tilde{\mathcal{V}})}^{2} \leq C \sum_{l \leq m}\left|\rho^{-a+l} v\right|_{H^{l}(\tilde{\mathcal{V}})}^{2} .
$$

Recall the multi-index $\alpha=\left(\alpha_{1}, \alpha_{2}\right)$ and the notation $\partial_{c}^{\alpha}$ from Definition 2.1. Then, the following lemma concerns the connection between the two types of weighted spaces in the $2 \mathrm{D}$ neighborhood $\mathcal{V}$ of a vertex $Q^{z}$ in the $r z$-plane (Lemmas 2.10 and 2.11 in [22]).

Lemma 2.7. Let $\mathcal{V} \subset \Omega$ be the neighborhood of a vertex $Q^{z}$ and $\rho$ be the distance to $Q^{z}$. Suppose $v \in \mathcal{K}_{a, 1}^{m}(\mathcal{V})$. Then, for $0 \leq l \leq m$ and $a \in \mathbb{R}$,

$$
C\left\|\rho^{-a+l} v\right\|_{H_{1}^{l}(\mathcal{V})}^{2} \leq\|v\|_{\mathcal{K}_{a, 1}^{m}(\mathcal{V})}^{2} \leq C \sum_{l \leq m}\left|\rho^{-a+l} v\right|_{H_{1}^{l}(\mathcal{V})}^{2} .
$$

The following two lemmas concern the local behavior of functions in weighted spaces near the vertices.

Lemma 2.8. Recall $\vartheta$ in Definition 2.2. In the neighhood $\mathcal{V}$ of a vertex $Q^{r}$ away from the $z$-axis, for any $v \in H_{+, 0}^{1}(\Omega)$ (resp. $v \in H_{-, 0}^{1}(\Omega)$ ), we have

$$
\left\|\vartheta^{-1} v\right\|_{L_{1}^{2}(\mathcal{V})} \leq C\|v\|_{H_{+}^{1}(\mathcal{V})}, \quad\left(\text { resp. }\left\|\vartheta^{-1} v\right\|_{L_{1}^{2}(\mathcal{V})} \leq C\|v\|_{H_{-}^{1}(\mathcal{V})}\right) .
$$

Proof. On $\mathcal{V}$, both $H_{+}^{1}$ and $H_{-}^{1}$ (resp. $L_{1}^{2}$ ) are equivalent to the usual Sobolev space $H^{1}$ (resp. $L^{2}$ ), since $r$ is bounded away from 0 . Therefore, it suffices to show for any $v \in H^{1}(\mathcal{V}) \cap\left\{\left.v\right|_{\Gamma_{1}}=0\right\}$,

$$
\left\|\vartheta^{-1} v\right\|_{L^{2}(\mathcal{V})} \leq C\|v\|_{H^{1}(\mathcal{V})} .
$$

However, the estimate in (13) is well known based on a local Poincaré inequality. See $[20,19,25,4]$.

Lemma 2.9. Recall $\vartheta$ in Definition 2.2. Let $v \in H_{0}^{1}(\tilde{\Omega})$ and let $\tilde{\mathcal{V}}=\left(\mathcal{V} \cup \Gamma_{\mathcal{V}}\right) \times$ $[0,2 \pi)$ be the $3 D$ neighborhood of a vertex $Q^{z}$ on the $z$-axis. Then,

$$
\left\|\vartheta^{-1} v\right\|_{L^{2}(\tilde{\mathcal{V}})} \leq C\|v\|_{H^{1}(\tilde{\mathcal{V}})} .
$$

Proof. $\tilde{\mathcal{V}}$ can be characterized in the spherical coordinates $(\rho, \theta, \phi)$ centered at $Q^{z}$ :

$$
\tilde{\mathcal{V}}=\left\{(\rho, \omega), 0<\rho<L / 2, \omega \in \omega_{Q^{z}}\right\},
$$

where $\omega_{Q^{z}} \subset S^{2}$ is the polygonal domain on the unit sphere $S^{2}$. Then, for any $v \in H^{1}(\tilde{\mathcal{V}}) \cap\left\{\left.v\right|_{\partial \tilde{\Omega}}=0\right\}$,

$$
|\nabla v|^{2}=v_{x}^{2}+v_{y}^{2}+v_{z}^{2}=v_{\rho}^{2}+\frac{v_{\phi}^{2}}{\rho^{2}}+\frac{v_{\theta}^{2}}{\rho^{2} \sin ^{2} \phi},
$$


and

$$
\int_{\omega_{Q} z} v^{2} d S \leq C \int_{\omega_{Q} z}\left(v_{\phi}^{2}+\frac{v_{\theta}^{2}}{\sin ^{2} \phi}\right) \sin \phi d \theta d \phi,
$$

which is just the Poincaré inequality on $\omega_{Q z}$ and $d S=\sin \phi d \theta d \phi$ is the volume element on $\omega_{Q^{z}}$. Thus, we obtain

$$
\begin{array}{r}
\int_{\tilde{\mathcal{V}}} \frac{v^{2}}{\rho^{2}} d x d y d z=\int_{0}^{L / 2} \int_{\omega_{Q} z} v^{2} d S d \rho \leq C \int_{0}^{L / 2} \int_{\omega_{Q} z}\left(v_{\rho}^{2}+\frac{v_{\phi}^{2}}{\rho^{2}}+\frac{v_{\theta}^{2}}{\rho^{2} \sin ^{2} \phi}\right) \rho^{2} d S d \rho \\
=C \int_{\tilde{\mathcal{V}}}|\nabla v|^{2} d x d y d z
\end{array}
$$

which completes the proof.

\section{Regularity AND Finite ELEMENT ANALYSIS}

The solution $u_{\beta}$ for equation (1) is well defined in weighted spaces (Corollary $2.5)$. When the $3 \mathrm{D}$ domain $\tilde{\Omega}$ is smooth, using the isomorphism in (10), we will have full regularity estimates in type I weighted spaces. These estimates will not hold when $\Omega$ is a general polygonal domain, since the regularity of $u_{\beta}$ is determined by the smoothness of $\Omega$ and by the operator $\mathcal{L}_{\beta}$. In this section, we show type II weighted spaces are suitable to study singular solutions. We derive high-order regularity estimates in these spaces for singular solutions and propose finite element algorithms approximating singular solutions in the optimal rate.

3.1. Regularity analysis. Before formulating our main regularity result (Theorem 3.5), we first need several local regality estimates. All these results are written for different values of the parameter $\beta$, namely, 0 or 1 .

Lemma 3.1. Near a vertex $Q^{r}$ away from the $z$-axis, there exists $\eta_{\beta}>0$, such that for any $0 \leq a_{\beta}<\eta_{\beta}$, if $f_{\beta} \in \mathcal{K}_{a_{\beta}-1,1}^{m}(\mathcal{V}), m \geq 0$, the solution $u_{\beta}$ of equation (11) satisfies

$$
\begin{gathered}
\left\|u_{0}\right\|_{\mathcal{K}_{a_{0}+1,1}^{m+2}(\mathcal{V} / 2)} \leq C\left(\left\|f_{0}\right\|_{\mathcal{K}_{a_{0}-1,1}^{m}(\mathcal{V})}+\left\|f_{0}\right\|_{H_{+, 0}^{1}(\Omega)^{\prime}}\right) \\
\left\|u_{1}\right\|_{\mathcal{K}_{a_{1}+1,1}^{m+2}(\mathcal{V} / 2)} \leq C\left(\left\|f_{1}\right\|_{\mathcal{K}_{a_{1}-1,1}^{m}(\mathcal{V})}+\left\|f_{1}\right\|_{H_{-, 0}^{1}(\Omega)^{\prime}}\right)
\end{gathered}
$$

Proof. We only show (14), since the proof of (15) follows similarly. We apply a localization argument. Let $\zeta$ be a smooth cutoff function, such that $\zeta=1$ on $\mathcal{V} / 2$ and $\zeta=0$ outside $\mathcal{V}$. Thus, $\zeta u_{0}$ has the Dirichlet boundary condition on $\mathcal{V}$. Then,

$$
-\left(\partial_{r}^{2}+r^{-1} \partial_{r}+\partial_{z}^{2}\right) \zeta u_{0}=\zeta f_{0}+g_{0} \text { in } \mathcal{V}
$$

where

$$
g_{0}=u_{0}\left(\partial_{r}^{2}+\partial_{z}^{2}\right) \zeta+2\left(\partial_{r} \zeta \partial_{r} u_{0}+\partial_{z} \zeta \partial_{z} u_{0}\right)+r^{-1} u_{0} \partial_{r} \zeta
$$

Note that the support of $g_{0}$ is within $\mathcal{V} \backslash \mathcal{V} / 2$ and $\mathcal{K}_{\mu, 1}^{m}=H^{m}$ on $\mathcal{V} \backslash \mathcal{V} / 2$. Therefore, using the interior regularity estimate in $H^{m}$ [14] and (12), we have

$$
\begin{array}{r}
\left\|g_{0}\right\|_{\mathcal{K}_{a_{0}-1,1}^{m}(\mathcal{V})}=\left\|g_{0}\right\|_{\mathcal{K}_{a_{0}-1,1}^{m}}(\mathcal{V} \backslash \mathcal{V} / 2) \\
\leq C\left\|u_{0}\right\|_{H^{m+1}(\mathcal{V} \backslash \mathcal{V} / 2)} \leq C\left(\left\|f_{0}\right\|_{H^{m}(\mathcal{V} \backslash \mathcal{V} / 4)}+\left\|g_{0}\right\|_{H^{m}(\mathcal{V} \backslash \mathcal{V} / 2)} \|_{H_{+, 0}^{1}(\Omega)^{\prime}}\right) \\
\leq C\left(\left\|f_{0}\right\|_{\mathcal{K}_{a_{0}-1,1}^{m}}(\mathcal{V} \backslash \mathcal{V} / 4)\right. \\
\left.\leq\left\|f_{0}\right\|_{H_{+, 0}^{1}(\Omega)^{\prime}}\right)
\end{array}
$$

By Corollary 2.5, equation (16) has a unique solution $\zeta u_{0} \in H_{+}^{1}(\mathcal{V})$. Since $r$ is bounded away from 0 on $\mathcal{V}, \zeta u_{0} \in H^{1}(\mathcal{V})$ and the space $\mathcal{K}_{\mu, 1}^{m}(\mathcal{V})$ is the same as the 
Kondrat'ev space $\mathcal{K}_{\mu}^{m}(\mathcal{V})$ (Definition 1.1 in [3]). In addition, the regularity of $\zeta u_{0}$ is determined by the principle part $-\partial_{r}^{2}-\partial_{z}^{2}$ of the operator in (16). Then, based on the $2 \mathrm{D}$ regularity estimates in the Kondrat'ev spaces for the Laplace operator $[18,24,16]$, for the solution $\zeta u_{0} \in H^{1}(\mathcal{V})$, there exists $\eta_{0}>0$, such that for any $0 \leq a_{0}<\eta_{0}$,

$$
\begin{aligned}
\left\|\zeta u_{0}\right\|_{\mathcal{K}_{a_{0}+1,1}^{m+2}(\mathcal{V})} \leq C\left\|\zeta f_{0}+g_{0}\right\|_{\mathcal{K}_{a_{0}-1,1}^{m}(\mathcal{V})} \leq & C\left(\left\|\zeta f_{0}\right\|_{\mathcal{K}_{a_{0}-1,1}^{m}(\mathcal{V})}+\left\|g_{0}\right\|_{\mathcal{K}_{a_{0}-1,1}^{m}(\mathcal{V})}\right) \\
\leq C\left(\left\|f_{0}\right\|_{\mathcal{K}_{a_{0}-1,1}^{m}}(\mathcal{V})\right. & \left.+\left\|f_{0}\right\|_{H_{+, 0}^{1}(\Omega)^{\prime}}\right) .
\end{aligned}
$$

The lemma is thus proved due to the definition of the function $\zeta$.

Remark 3.2. Near the vertex $Q^{r}$, since $r$ is away from 0 , the principle part of the operator $\mathcal{L}_{\beta}$ coincides with the Laplace operator $-\partial_{r}^{2}-\partial_{z}^{2}$. Therefore, the regularity index $\eta_{\beta}$ is the same for $\beta=0$ and $\beta=1$. Let $\omega$ be the interior angle at $Q^{r}$. Then,

$$
\eta_{0}=\eta_{1}=\pi / \omega
$$

which is the first eigenvalue of the operator pencil associated with the Laplace operator $[16,18,19,24]$.

Define $\tilde{u}_{\beta}(x, y, z):=\frac{1}{\sqrt{2 \pi}} u_{\beta}(r, z) e^{i \beta \theta}$ and $\tilde{f}_{\beta}(x, y, z):=\frac{1}{\sqrt{2 \pi}} f_{\beta}(r, z) e^{i \beta \theta}$. Then, we see that, by the change of variables, $\tilde{u}_{\beta}$ satisfies the $3 \mathrm{D}$ Poisson equation,

$$
-\Delta \tilde{u}_{\beta}=\tilde{f}_{\beta} \quad \text { in } \quad \tilde{\Omega} \quad \tilde{u}_{\beta}=0 \quad \text { on } \quad \partial \tilde{\Omega}, \quad \beta=0,1 .
$$

In addition, its Fourier coefficients

$$
\mathcal{F}_{k}\left(\tilde{u}_{\beta}\right)=\left\{\begin{array}{l}
u_{\beta} \text { if } k=\beta \\
0 \text { if } k \neq \beta
\end{array}\right.
$$

We now give a regularity estimate for $\tilde{u}_{\beta}$ near a vertex on the $z$-axis.

Lemma 3.3. For a vertex $Q^{z}$ on the $z$-axis, there is $\eta_{\beta}>0$ for all $m \geq 0$, such that for any $0 \leq a_{\beta}<\eta_{\beta}$, the solution $\tilde{u}_{\beta}$ of equation (18) satisfies

$$
\begin{aligned}
& \left(\sum_{|\alpha| \leq m+2}\left\|\vartheta^{-a_{\beta}-1+|\alpha|} \partial^{\alpha} \tilde{u}_{\beta}\right\|_{L^{2}(\tilde{\mathcal{V}} / 2)}^{2}\right)^{1 / 2} \\
& \leq C\left(\left(\sum_{|\alpha| \leq m}\left\|\vartheta^{-a_{\beta}+1+|\alpha|} \partial^{\alpha} \tilde{f}_{\beta}\right\|_{L^{2}(\tilde{\mathcal{V}})}^{2}\right)^{1 / 2}+\left\|\tilde{f}_{\beta}\right\|_{H^{-1}(\tilde{\Omega})}\right) .
\end{aligned}
$$

Proof. We use a localization augment similar to the one in Lemma 3.1. Let $\zeta$ be a smooth cutoff function, such that $\zeta=1$ on $\tilde{\mathcal{V}} / 2$ and $\zeta=0$ outside $\tilde{\mathcal{V}}$. Then,

$$
-\Delta \zeta \tilde{u}_{\beta}=\zeta \tilde{f}_{\beta}+\tilde{g}_{\beta},
$$

where

$$
\tilde{g}_{\beta}=\tilde{u}_{\beta} \Delta \zeta+2 \partial_{x} \tilde{u}_{\beta} \partial_{x} \zeta+2 \partial_{y} \tilde{u}_{\beta} \partial_{y} \zeta+2 \partial_{z} \tilde{u}_{\beta} \partial_{z} \zeta .
$$

Since $\tilde{g}_{\beta}$ vanishes near $Q^{z}$, using the well-posedness of the Poisson problem (18), the usual interior regularity estimate [14], and the expression of $\tilde{g}_{\beta}$ above, we have

$$
\begin{aligned}
& \left(\sum_{|\alpha| \leq m}\left\|\vartheta^{-a_{\beta}+1+|\alpha|} \partial^{\alpha} \tilde{g}_{\beta}\right\|_{L^{2}(\tilde{\mathcal{V}})}^{2}\right)^{1 / 2} \leq C\left(\left\|\tilde{u}_{\beta}\right\|_{H^{m+1}(\tilde{\mathcal{V}} \backslash \tilde{\mathcal{V}} / 2)}\right) \\
& \leq C\left(\left\|\tilde{f}_{\beta}\right\|_{H^{m-1}(\tilde{\mathcal{V}} \backslash \tilde{\mathcal{V}} / 4)}+\left\|\tilde{f}_{\beta}\right\|_{H^{-1}(\tilde{\Omega})}\right) \\
& \leq C\left(\left(\sum_{|\alpha| \leq m}\left\|\vartheta^{-a_{\beta}+1+|\alpha|} \partial^{\alpha} \tilde{f}_{\beta}\right\|_{L^{2}(\tilde{\mathcal{V}})}^{2}\right)^{1 / 2}+\left\|\tilde{f}_{\beta}\right\|_{H^{-1}(\tilde{\Omega})}\right) .
\end{aligned}
$$


Based on the 3D regularity estimates in the Kondrat'ev spaces for equation (19) (Theorem 1.2 in [2]) and (20), there exists $\eta_{\beta}>0$, such that for any $0 \leq a_{\beta}<\eta_{\beta}$, the solution $\zeta \tilde{u}_{\beta}$ satisfies

$$
\begin{aligned}
& \left(\sum_{|\alpha| \leq m+2}\left\|\vartheta^{-a_{\beta}-1+|\alpha|} \partial^{\alpha}\left(\zeta \tilde{u}_{\beta}\right)\right\|_{L^{2}(\tilde{\mathcal{V}})}^{2}\right)^{1 / 2} \\
& \leq C\left(\sum_{|\alpha| \leq m}\left\|\vartheta^{-a_{\beta}+1+|\alpha|} \partial^{\alpha}\left(\zeta \tilde{f}_{\beta}+\tilde{g}_{\beta}\right)\right\|_{L^{2}(\tilde{\mathcal{V}})}^{2}\right)^{1 / 2} \\
& \leq C\left(\left(\sum_{|\alpha| \leq m}\left\|\vartheta^{-a_{\beta}+1+|\alpha|} \partial^{\alpha}\left(\zeta \tilde{f}_{\beta}\right)\right\|_{L^{2}(\tilde{\mathcal{V}})}^{2}\right)^{1 / 2}+\left(\sum_{|\alpha| \leq m}\left\|\vartheta^{-a_{\beta}+1+|\alpha|} \partial^{\alpha} \tilde{g}_{\beta}\right\|_{L^{2}(\tilde{\mathcal{V}})}^{2}\right)^{1 / 2}\right) \\
& \leq C\left(\left(\sum_{|\alpha| \leq m}\left\|\vartheta^{-a_{\beta}+1+|\alpha|} \partial^{\alpha} \tilde{f}_{\beta}\right\|_{L^{2}(\tilde{\mathcal{V}})}^{2}\right)^{1 / 2}+\left\|\tilde{f}_{\beta}\right\|_{H^{-1}(\tilde{\Omega})}\right) .
\end{aligned}
$$

The lemma is thus proved due to the definition of $\zeta$.

Remark 3.4. Different from the situation in Lemma 3.1 and Remak 3.2, near the vertex $Q^{z}$, the index $\eta_{\beta}$ is determined by the operator pencil of the 3D Laplace operator in a bounded region on the unit sphere $[16,18]$. To be more precise, in the neighborhood $\tilde{\mathcal{V}}$ of $Q^{z}$, let $\omega$ be the projection of $\tilde{\mathcal{V}}$ on the unit sphere. We write equation (18) in the polar coordinations, $-\Delta \tilde{u}_{\beta}=-\rho^{-2}\left(\left(\rho \partial_{\rho}\right)^{2}+\rho \partial_{\rho}+\Delta^{\prime}\right) \tilde{u}_{\beta}$, where $\Delta^{\prime}$ is the Laplace-Beltrami operator on $\omega$,

$$
\Delta^{\prime}=\cot \phi \partial_{\phi}+\partial_{\phi}^{2}+\sin ^{-2} \phi \partial_{\theta}^{2} .
$$

For any $v(r, z) \in L_{1}^{2}(\mathcal{V})$, define $\tilde{v}_{\beta}:=\frac{1}{\sqrt{2 \pi}} v e^{i \beta \theta}$. The smallest eigenvalue $\lambda_{\beta}$ of $\Delta^{\prime}$ with respect to $\tilde{v}_{\beta}$ is given by

$$
\begin{gathered}
\lambda_{0} \tilde{v}_{0}=-\Delta^{\prime} \tilde{v}_{0}=-\left(\cot \phi \partial_{\phi}+\partial_{\phi}^{2}\right) \tilde{v}_{0}, \\
\lambda_{1} \tilde{v}_{1}=-\Delta^{\prime} \tilde{v}_{1}=-\left(\cot \phi \partial_{\phi}+\partial_{\phi}^{2}-\sin ^{-2} \phi\right) \tilde{v}_{1},
\end{gathered}
$$

which is determined by the associated Legendre's differential equation [13, 27]. Then, the explicit formula for the index $\eta_{\beta}$ near $Q^{z}$ is

$$
\eta_{\beta}=\sqrt{\lambda_{\beta}+1 / 4}
$$

In contrast to the case near $Q^{r}$ in (17), $\eta_{0}$ and $\eta_{1}$ may be different near $Q^{z}$, since $\lambda_{\beta}$ are eigenvalues associated with different differential operators.

Combining the local estimates in the lemmas above, we derive the global regularity estimate for equation (1).

Theorem 3.5. Let $u_{0} \in H_{+, 0}^{1}(\Omega)$ and $u_{1} \in H_{-, 0}^{1}(\Omega)$ be the solutions of equation (1) with $\beta=0$ and 1 , respectively. For $m \geq 0$, there exist $\Upsilon_{0}>0$ and $\Upsilon_{1}>0$, such that for any $0 \leq a_{0}<\Upsilon_{0}$ and $0 \leq a_{1}<\Upsilon_{1}$, we have

$$
\begin{gathered}
\left\|u_{0}\right\|_{\mathcal{K}_{a_{0}+1,1}^{m+2}(\Omega)} \leq C\left\|f_{0}\right\|_{\mathcal{K}_{a_{0}-1,+}^{m}(\Omega)}, \\
\left\|\vartheta^{-a_{1}} r^{-1} u_{1}\right\|_{L_{1}^{2}(\Omega)}+\left\|u_{1}\right\|_{\mathcal{K}_{a_{1}+1,1}^{m+2}(\Omega)} \leq C\left\|f_{1}\right\|_{\mathcal{K}_{a_{1}-1,-}^{m}(\Omega)},
\end{gathered}
$$

given that $f_{0} \in \mathcal{K}_{a-1,+}^{m}(\Omega)$ and $f_{1} \in \mathcal{K}_{a-1,-}^{m}(\Omega)$.

Proof. Let $\eta_{\beta}^{i}$ be the upper bound of the parameter $a_{\beta}$ for the $i$ th vertex $Q_{i}$ in Lemmas 3.1 and 3.3. Define $\Upsilon_{0}=\min _{i}\left(\eta_{0}^{i}\right)$ and $\Upsilon_{1}=\min _{i}\left(\eta_{1}^{i}\right)$. We only show (23), since the proof for (22) follows similarly. 
Recall that the weighted space $\mathcal{K}_{a, 1}^{m}$ (resp., $\mathcal{K}_{a,+}^{m}$ and $\mathcal{K}_{a,-}^{m}$ ) is equivalent to the weighted space $H_{1}^{m}$ (resp., $H_{+}^{m}$ and $H_{-}^{m}$ ) in a subdomain $\Omega_{\text {sub }} \subset \Omega$ that is away from the vertex set. Based on the isomorphism in (10), the well-posedness and the usual interior regularity estimate for the 3D Poisson equation (18), we have

$$
\begin{array}{r}
\left\|\vartheta^{-a_{1}} r^{-1} u_{1}\right\|_{L_{1}^{2}\left(\Omega_{s u b}\right)}+\left\|u_{1}\right\|_{\mathcal{K}_{a_{1}+1,1}^{m+2}\left(\Omega_{s u b}\right)} \leq C\left\|\tilde{f}_{1}\right\|_{H^{m}\left(\tilde{\Omega}^{\prime}\right)}+\left\|\tilde{f}_{1}\right\|_{H^{-1}(\tilde{\Omega})} \\
\leq C\left(\left\|f_{1}\right\|_{\mathcal{K}_{a_{1}-1,-}^{m}\left(\Omega^{\prime}\right)}+\left\|f_{1}\right\|_{\mathcal{K}_{a_{1}-1,-}^{0}(\Omega)}\right)
\end{array}
$$

where $\Omega_{\text {sub }} \subset \Omega^{\prime} \subset \Omega$ such that $\Omega^{\prime}$ is away from the vertex set, and $\tilde{\Omega}^{\prime}=\left(\Omega^{\prime} \cup\right.$ $\left.\left(\bar{\Omega}^{\prime} \cap\{r=0\}\right)\right) \times[0,2 \pi)$ is from the rotation of $\Omega^{\prime}$ about the $z$-axis.

Let $\mathcal{V}$ be the neighborhood of a vertex $Q^{r}$ away from the $z$-axis. By Lemma 3.1, the fact that $r$ is bounded away from 0 on $\mathcal{V}$, Lemma 2.8, and $a_{1} \geq 0$, we have

$$
\begin{aligned}
& \left\|\vartheta^{-a_{1}} r^{-1} u_{1}\right\|_{L_{1}^{2}(\mathcal{V} / 2)}+\left\|u_{1}\right\|_{\mathcal{K}_{a_{1}+1,1}^{m+2}(\mathcal{V} / 2)} \leq C\left(\left\|f_{1}\right\|_{\mathcal{K}_{a_{1}-1,1}^{m}(\mathcal{V})}+\left\|f_{1}\right\|_{H_{-, 0}^{1}(\Omega)^{\prime}}\right) \\
& =C\left(\left\|f_{1}\right\|_{\mathcal{K}_{a_{1}-1,1}^{m}(\mathcal{V})}+\sup _{v \in H_{-, 0}^{1}(\Omega), v \neq 0}\left(f_{1}, v\right)_{L_{1}^{2}} /\|v\|_{H_{-, 0}^{1}(\Omega)}\right) \\
& \leq C\left(\left\|f_{1}\right\|_{\mathcal{K}_{a_{1}-1,-}^{m}(\mathcal{V})}+\left\|f_{1}\right\|_{\mathcal{K}_{a_{1}-1,-}^{0}(\Omega)}\right) .
\end{aligned}
$$

Now, let $\mathcal{V}$ be the small neighborhood of a vertex $Q^{z}$ on the $z$-axis. By Lemma 2.7 , we first have for any $0 \leq l \leq m$,

$$
\left\|\vartheta^{1-a_{1}+l} f_{1}\right\|_{H_{1}^{l}(\mathcal{V})} \leq C\left\|f_{1}\right\|_{\mathcal{K}_{a_{1}-1,1}^{m}(\mathcal{V})} .
$$

Then, for $f_{1} \in \mathcal{K}_{a_{1}-1,-}^{m}(\mathcal{V}),(26)$ and the condition in $(9)$

$$
\int_{\Omega}\left(\partial_{r}^{2 i}\left(\vartheta^{1-a_{1}+l} f_{1}\right)\right)^{2} r^{-1} d r d z<\infty, \quad 0 \leq i \leq(l-1) / 2
$$

lead to $\vartheta^{1-a_{1}+l} f_{1} \in H_{-}^{l}(\mathcal{V})$. Then, by Lemma 2.6, the isomorphism in $(10)$, and the definitions of the weighted spaces in (7) and (9), we have

$$
\begin{aligned}
& \sum_{|\alpha| \leq m}\left\|\vartheta^{1-a_{1}+|\alpha|} \partial^{\alpha} \tilde{f}_{1}\right\|_{L^{2}(\tilde{\mathcal{V}})}^{2} \leq C \sum_{0 \leq l \leq m}\left\|\vartheta^{1-a_{1}+l} \tilde{f}_{1}\right\|_{H^{l}(\tilde{\mathcal{V}})}^{2} \\
& \leq C \sum_{0 \leq l \leq m}\left\|\vartheta^{1-a_{1}+l} f_{1}\right\|_{H_{-}^{l}(\mathcal{V})}^{2} \leq C\left\|f_{1}\right\|_{\mathcal{K}_{a_{1}-1,-}^{m}}^{2}(\mathcal{V}) \cdot
\end{aligned}
$$

Then, by Lemma 2.7, the isomorphism in (10), Lemma 2.6, Lemma 3.3, Lemma $2.9,(27)$, and $a_{1} \geq 0$, we have

$$
\begin{aligned}
& \left\|\vartheta^{-a_{1}} r^{-1} u_{1}\right\|_{L_{1}^{2}(\mathcal{V} / 2)}+\left\|u_{1}\right\|_{\mathcal{K}_{a_{1}+1,1}^{m+2}(\mathcal{V} / 2)} \\
& \leq C\left(\left\|\vartheta^{-a_{1}} r^{-1} u_{1}\right\|_{L_{1}^{2}(\mathcal{V} / 2)}+\left(\sum_{l \leq m+2}\left\|\vartheta^{-a_{1}-1+l} u_{1}\right\|_{H_{1}^{l}(\mathcal{V} / 2)}^{2}\right)^{1 / 2}\right) \\
& \leq C\left(\sum_{l \leq m+2}\left\|\vartheta^{-a_{1}-1+l} \tilde{u}_{1}\right\|_{H^{l}(\tilde{\mathcal{V}} / 2)}^{2}\right)^{1 / 2} \\
& \leq C\left(\left(\sum_{|\alpha| \leq m}\left\|\vartheta^{-a_{1}+1+|\alpha|} \partial^{\alpha} \tilde{f}_{1}\right\|_{L^{2}(\tilde{\mathcal{V}})}^{2}\right)^{1 / 2}+\sup _{v \in H_{0}^{1}(\tilde{\Omega}), v \neq 0}\left(\tilde{f}_{1}, v\right)_{L^{2}(\tilde{\Omega})} /\|v\|_{H^{1}(\tilde{\Omega})}\right) \\
& \leq C\left(\left(\sum_{|\alpha| \leq m}\left\|\vartheta^{-a_{1}+1+|\alpha|} \partial^{\alpha} \tilde{f}_{1}\right\|_{L^{2}(\tilde{\mathcal{V}})}^{2}\right)^{1 / 2}+\left\|\vartheta^{-a_{1}+1} \tilde{f}_{1}\right\|_{L^{2}(\tilde{\Omega})}\right) \\
(28) & \leq C\left(\left\|f_{1}\right\|_{\mathcal{K}_{a_{1}-1,-}^{m}(\mathcal{V})}+\left\|f_{1}\right\|_{\mathcal{K}_{a_{1}-1,-}^{0}(\Omega)}\right) .
\end{aligned}
$$

Then, the proof is completed by combining (24), (25), and (28). 
Remark 3.6. We used the global parameter $\Upsilon_{\beta}$ in Theorem 3.5 in order to simplify the exposition. The proof in fact shows that Theorem 3.5 holds as long as for the $i$ th vertex $Q_{i}$, we choose the parameter $0 \leq a_{\beta}<\eta_{\beta}^{i}$, where $\eta_{\beta}^{i}$ is determined as in Remarks 3.2 and 3.4. This will result in the analogue of Theorem 3.5 in Kondrat'ev-type spaces with vector subindices, in which the function $\vartheta$ is allowed to have different exponents near different vertices to capture the local behavior of the solution. See [24] for such a presentation for elliptic equations.

3.2. Finite element analysis. Let $\mathcal{T}_{n}=\left\{T_{i}\right\}$ be a triangulation of $\Omega$ with shaperegular triangles $T_{i}$. Denote by $\mathbb{V}_{n}^{\beta}$ the continuous Lagrange finite element space of order $m$ with the corresponding Dirichlet boundary conditions described in (2) and (3), respectively. Then, we have [21]

$$
\mathbb{V}_{n}^{0} \subset H_{+, 0}^{1}(\Omega) \text { and } \mathbb{V}_{n}^{1} \subset H_{-, 0}^{1}(\Omega)
$$

In turn, the finite element solution $u_{n}^{\beta} \in \mathbb{V}_{n}^{\beta}$ for equation (11) is defined by

$$
a_{\beta}\left(u_{n}^{\beta}, v_{n}^{\beta}\right)=\int_{\Omega} f_{\beta} v_{n}^{\beta} r d r d z, \quad \forall v_{n}^{\beta} \in \mathbb{V}_{n}^{\beta} .
$$

We denote a point in the $r z$-plane by $\mathbf{x}=(r, z)$. Let $\Pi: \mathcal{C}^{0}(\Omega) \rightarrow \mathbb{V}_{k}^{\beta}$ be the usual nodal interpolation operator, such that $\Pi v\left(\mathbf{x}_{i}\right)=v\left(\mathbf{x}_{i}\right)$, where $\mathbf{x}_{i}$ is the $i$ th node in the triangulation. Note that we use the same notation for the interpolation operators into different spaces $\mathbb{V}_{n}^{\beta}, \beta=0$ and 1 . In the text below, by a triangle $T$, we mean the closed set including both the interior and boundary of the triangle.

We first recall two approximation results from Lemmas 6.1, 6.2 and 6.3 in [26], which are based on embedding theorems in weighted spaces.

Lemma 3.7. Let $\mathbf{x}_{i}$ be a node in the triangulation, which is on the $z$-axis. Let $K_{i}$ be the union of triangles that contain $\mathbf{x}_{i}$. Then, for $v \in H_{1}^{m+1}\left(K_{i}\right)$, we have

$$
|v-\Pi v|_{H_{1}^{1}\left(K_{i}\right)} \leq C h_{K_{i}}^{m}|v|_{H_{1}^{m+1}\left(K_{i}\right)}
$$

for $v \in H_{1}^{m+1}\left(K_{i}\right) \cap H_{-}^{1}(\Omega)$,

$$
\left\|r^{-1}(v-\Pi v)\right\|_{L_{1}^{2}\left(K_{i}\right)} \leq C h_{K_{i}}^{m}|v|_{H_{1}^{m+1}\left(K_{i}\right)},
$$

where $h_{K_{i}}$ denotes the diameter of $K_{i}$ and $m \geq 1$.

Lemma 3.8. Let $T \in \mathcal{T}_{n}$ be a triangle that does not intersect the $z$-axis. Suppose $\min _{\mathbf{x} \in T} r(\mathbf{x}) \geq C h_{T}$. Then, for $v \in H_{1}^{m+1}(T)$, we have

$$
\begin{aligned}
|v-\Pi v|_{H_{1}^{1}(T)} & \leq C h_{T}^{m}|v|_{H_{1}^{m+1}(T)}, \\
\left\|r^{-1}(v-\Pi v)\right\|_{L_{1}^{2}(T)} & \leq C h_{T}^{m}|v|_{H_{1}^{m+1}(T)},
\end{aligned}
$$

where $h_{T}$ denotes the diameter of $T$ and $m \geq 1$.

Remark 3.9. By Lemma 3.7 and Lemma 3.8, one can derive the convergence result for the finite element solution in (30) on quasi-uniform meshes

$$
\left\|u_{0}-u_{n}^{0}\right\|_{H_{+}^{1}(\Omega)} \leq C h^{m}\left\|u_{0}\right\|_{H_{+}^{m+1}(\Omega)} \quad \text { and } \quad\left\|u_{1}-u_{n}^{1}\right\|_{H_{-}^{1}(\Omega)} \leq C h^{m}\left\|u_{1}\right\|_{H_{-}^{m+1}(\Omega)},
$$

given that $u_{\beta}$ is sufficiently regular and $h$ is the mesh size. As discussed above, the regularity of the solution, however, depends on the differential operator $\mathcal{L}_{\beta}$ and the domain $\Omega$. When the solution $u_{\beta}$ is singular (e.g., $u_{\beta} \notin H_{1}^{2}(\Omega)$ ), the linear finite element method on the quasi-uniform mesh only gives a suboptimal convergence rate $[6]$. 
Based on our regularity estimates in Theorem 3.5 and Remark 3.6, we use the following process to generate a sequence of graded triangulations approximating the singular solutions of equation (1).

Definition 3.10. (The $\kappa$-refinement). Let $\kappa \in(0,1 / 2]$ be the grading parameter and $\mathcal{T}$ be a triangulation of $\Omega$ such that no two vertices of $\Omega$ belong to the same triangle of $\mathcal{T}$. Then the $\kappa$-refinement of $\mathcal{T}$, denoted by $\kappa(\mathcal{T})$, is obtained by dividing each edge $A B$ of $\mathcal{T}$ into two parts as follows. If neither $A$ nor $B$ is in the vertex set $\mathcal{Q}$, then we divide $A B$ into two equal parts. Otherwise, if $A \in \mathcal{Q}$, we divide $A B$ into $A C$ and $C B$ such that $|A C|=\kappa|A B|$. This will divide each triangle of $\mathcal{T}$ into four triangles and leads to a finer mesh. Suppose the initial mesh $\mathcal{T}_{0}$ satisfies the above conditions. Then the $n$th level mesh with $\kappa$-refinement is obtained recursively by $\mathcal{T}_{n}=\kappa\left(\mathcal{T}_{n-1}\right), n=1,2, \ldots$

This graded process was proposed in [23] for the linear finite element method approximating the axisymmetric Poisson equation. We here generalize it to highorder finite element methods for the new equation (1).

We need the following notation to carry out the analysis on graded meshes. Let $n$ be the number of $\kappa$-refinements of the domain. Thus, the final triangulation is $\mathcal{T}_{n}$. Let $\mathbb{T}_{i, j} \subset \mathcal{T}_{j}, j \leq n$, be the union of triangles in $\mathcal{T}_{j}$ that contain the vertex $Q_{i} \in \mathcal{Q}$. Note that $\mathbb{T}_{i, j} \subset \mathbb{T}_{i, l}$ for $j \geq l$ and $\cup_{i} \mathbb{T}_{i, j}$ occupies the neighborhood of the vertex set $\mathcal{Q}$ in the triangulation $\mathcal{T}_{j}$. Recall the regularity estimate for the solution and the parameter $\Upsilon_{\beta}$ in Theorem 3.5. We choose the grading parameter in the $\kappa$-refinement, for different values of $\beta$,

$$
\kappa_{\beta}=\min \left(1 / 2,2^{-m / a_{\beta}}\right), \quad \text { for any } 0<a_{\beta}<\Upsilon_{\beta},
$$

where $m \geq 1$ is the degree of piecewise polynomials in the finite element space $\mathbb{V}_{n}^{\beta}$. Then, based on analysis on $\mathcal{T}_{n} \backslash \cup_{i} \mathbb{T}_{i, 0}$, on $\cup_{i} \mathbb{T}_{i, j-1} \backslash \cup_{i} \mathbb{T}_{i, j}$, and on $\mathbb{T}_{i, n}$, our error estimates are summarized in the following lemmas.

Lemma 3.11. For $\kappa_{\beta}$ defined in (35), let $U \subset \mathcal{T}_{n}$ be the union of triangles that intersect $\mathcal{T}_{n} \backslash \cup_{i} \mathbb{T}_{i, 0}$. Then,

$$
\begin{aligned}
\left\|u_{0}-\Pi u_{n}^{0}\right\|_{H_{+}^{1}\left(\mathcal{T}_{n} \backslash \cup_{i} \mathbb{T}_{i, 0}\right)} & \leq C 2^{-n m}\left\|u_{0}\right\|_{H_{1}^{m+1}(U)} \\
\left\|u_{1}-\Pi u_{n}^{1}\right\|_{H_{-}^{1}\left(\mathcal{T}_{n} \backslash \cup_{i} \mathbb{T}_{i, 0}\right)} & \leq C 2^{-n m}\left\|u_{1}\right\|_{H_{1}^{m+1}(U)} .
\end{aligned}
$$

Proof. Assume $U$ is away from the vertices of the domain (this is true when $n>2$ ). Then, based on Definition 3.10, the mesh size on $U$ is $\mathcal{O}\left(2^{-n}\right)$. Summing up the estimates in (31), (32), (33), and (34) completes the proof.

For the estimates on $\mathbb{T}_{i, 0}$, the union of initial triangles containing the vertex $Q_{i}$, we consider the new coordinate system that is a simple translation of the old $r z$-coordinate system, now with $Q_{i}$ at the origin. Then, for a subset $G \subset \mathbb{T}_{i, 0}$ and $0<\lambda<1$, we define the dilations of $G$ and of a function as follows, for $(r, z) \in G$,

$$
G_{\lambda}:=G / \lambda=\left\{\left(r_{\lambda}, z_{\lambda}\right)=\left(\lambda^{-1} r, \lambda^{-1} z\right)\right\}, \quad v_{\lambda}\left(r_{\lambda}, z_{\lambda}\right):=v(r, z) .
$$

Then, we have the following scaling estimates from Lemma 4.5, [22].

Lemma 3.12. Suppose $G_{\lambda}$ is in the neighborhood $\mathcal{V}$ of $Q_{i}$. If $Q_{i}$ is on the z-axis,

$$
\begin{aligned}
\left\|v_{\lambda}\right\|_{\mathcal{K}_{a, 1}^{m}\left(G_{\lambda}\right)} & =\lambda^{a-3 / 2}\|v\|_{\mathcal{K}_{a, 1}^{m}(G)}, \\
\left\|r_{\lambda}^{-1} v_{\lambda}\right\|_{L_{1}^{2}\left(G_{\lambda}\right)} & =\lambda^{-1 / 2}\left\|r^{-1} v\right\|_{L_{1}^{2}(G)} ;
\end{aligned}
$$


if $Q_{i}$ is not on the z-axis,

$$
C \lambda^{a-1}\|v\|_{\mathcal{K}_{a, 1}^{m}(G)} \leq\left\|v_{\lambda}\right\|_{\mathcal{K}_{a, 1}^{m}\left(G_{\lambda}\right)} \leq C \lambda^{a-1}\|v\|_{\mathcal{K}_{a, 1}^{m}(G)} .
$$

Now, we are ready to give estimates on the region $\mathbb{T}_{i, j-1} \backslash \mathbb{T}_{i, j}$.

Lemma 3.13. Let $U \subset \mathcal{T}_{n}$ be the union of triangles that intersect $G:=\mathbb{T}_{i, j-1} \backslash \mathbb{T}_{i, j}$. Suppose $G \subset \mathcal{V}$. Let $h$ be the mesh size on $U$ and $\xi=\sup _{\mathbf{x} \in G} \vartheta(\mathbf{x})$. Let $a \geq 0$. Then, for $v \in \mathcal{K}_{a+1,1}^{m+1}(U)$,

$$
\|v-\Pi v\|_{H_{+}^{1}(G)} \leq C \xi^{a}(h / \xi)^{m}\|v\|_{\mathcal{K}_{a+1,1}^{m+1}(U)},
$$

and for $v \in H_{-}^{1}(U) \cap \mathcal{K}_{a+1,1}^{m+1}(U)$,

$$
\|v-\Pi v\|_{H_{-}^{1}(G)} \leq C \xi^{a}(h / \xi)^{m}\|v\|_{\mathcal{K}_{a+1,1}^{m+1}(U)} .
$$

Proof. Recall the new coordinate system with $Q_{i}$ as the origin. Recall the dilations in (36) and the parameter $L$ in Definition 2.2. Note that $(\Pi v)_{\lambda}=\Pi\left(v_{\lambda}\right)$. Then, we choose $\lambda=2 \xi / L$, such that $G_{\lambda} \subset \mathcal{V}$.

If $Q_{i}$ is on the $z$-axis, by Lemma 3.12, the definitions of the weighted spaces, (31), and (32), we have

$$
\begin{aligned}
& \left\|r^{-1}(v-\Pi v)\right\|_{L_{1}^{2}(G)}+\|v-\Pi v\|_{H_{1}^{1}(G)} \\
& \leq C\left\|r^{-1}(v-\Pi v)\right\|_{L_{1}^{2}(G)}+\|v-\Pi v\|_{\mathcal{K}_{1,1}^{1}(G)} \\
& =\lambda^{1 / 2}\left(\left\|r_{\lambda}^{-1}\left(v_{\lambda}-\Pi\left(v_{\lambda}\right)\right)\right\|_{L_{1}^{2}\left(G_{\lambda}\right)}+\left\|v_{\lambda}-\Pi\left(v_{\lambda}\right)\right\|_{\mathcal{K}_{1,1}^{1}\left(G_{\lambda}\right)}\right) \\
& \leq C \lambda^{1 / 2}\left(\left\|r_{\lambda}^{-1}\left(v_{\lambda}-\Pi\left(v_{\lambda}\right)\right)\right\|_{L_{1}^{2}\left(G_{\lambda}\right)}+\left\|v_{\lambda}-\Pi\left(v_{\lambda}\right)\right\|_{H_{1}^{1}\left(G_{\lambda}\right)}\right) \\
& \leq C \lambda^{1 / 2}(h / \lambda)^{m}\left\|v_{\lambda}\right\|_{H_{1}^{m+1}\left(U_{\lambda}\right)} \leq C \lambda^{1 / 2}(h / \lambda)^{m}\left\|v_{\lambda}\right\|_{\mathcal{K}_{1,1}^{m+1}\left(U_{\lambda}\right)} \\
& \leq C(h / \xi)^{m}\|v\|_{\mathcal{K}_{1,1}^{m+1}(U)} \leq C \xi^{a}(h / \xi)^{m}\|v\|_{\mathcal{K}_{a+1,1}^{m+1}(U)} .
\end{aligned}
$$

If $Q_{i}$ is not on the $z$-axis, the proof is similar. With the corresponding estimate in Lemma 3.12, the definitions of the weighted spaces, (33), and (34), we have

$$
\begin{aligned}
& \left\|r^{-1}(v-\Pi v)\right\|_{L_{1}^{2}(G)}+\|v-\Pi v\|_{H_{1}^{1}(G)} \\
& \leq C\left\|r^{-1}(v-\Pi v)\right\|_{L_{1}^{2}(G)}+\|v-\Pi v\|_{\mathcal{K}_{1,1}^{1}(G)} \leq C\|v-\Pi v\|_{\mathcal{K}_{1,1}^{1}(G)} \\
& \leq C\left\|v_{\lambda}-\Pi\left(v_{\lambda}\right)\right\|_{\mathcal{K}_{1,1}^{1}\left(G_{\lambda}\right)} \leq C\left\|v_{\lambda}-\Pi\left(v_{\lambda}\right)\right\|_{H_{1}^{1}\left(G_{\lambda}\right)} \leq C(h / \lambda)^{m}\left\|v_{\lambda}\right\|_{H_{1}^{m+1}\left(U_{\lambda}\right)} \\
& \leq C(h / \lambda)^{m}\left\|v_{\lambda}\right\|_{\mathcal{K}_{1,1}^{m+1}\left(U_{\lambda}\right)} \leq C(h / \xi)^{m}\|v\|_{\mathcal{K}_{1,1}^{m+1}(U)} \leq C \xi^{a}(h / \xi)^{m}\|v\|_{\mathcal{K}_{a+1,1}^{m+1}(U)} .
\end{aligned}
$$

Thus, the estimate in (38) is proved.

The estimate in (37) follows similarly using Lemma 3.12, the definitions of the weighted spaces, (31), and (33).

Lemma 3.14. For $\kappa_{\beta}$ defined in (35), let $U \subset \mathcal{T}_{n}$ be the union of triangles that intersect $G:=\mathbb{T}_{i, j-1} \backslash \mathbb{T}_{i, j}$. Then, for $0<a_{\beta}<\Upsilon_{\beta}$,

$$
\begin{aligned}
\left\|u_{0}-\Pi u_{0}\right\|_{H_{+}^{1}(G)} \leq C 2^{-n m}\left\|u_{0}\right\|_{\mathcal{K}_{a_{0}+1,1}^{m+1}(U)}, \\
\left\|u_{1}-\Pi u_{1}\right\|_{H_{-}^{1}(G)} \leq C 2^{-n m}\left\|u_{1}\right\|_{\mathcal{K}_{a_{1}+1,1}^{m+1}(U)} .
\end{aligned}
$$

Proof. Definition 3.10 shows that the mesh size on $U$ is $\mathcal{O}\left(\kappa_{\beta}^{j-1} 2^{j-1-n}\right)$. Using the notation of Lemma 3.13 , we have $\xi=\mathcal{O}\left(\kappa_{\beta}^{j-1}\right)$ on $\mathbb{T}_{i, j-1} \backslash \mathbb{T}_{i, j}$. Therefore, using 
Lemma 3.13, we have

$$
\begin{aligned}
& \left.\| u_{1}-\Pi u_{1}\right)\left\|_{H_{-}^{1}(G)} \leq C \kappa_{1}^{(j-1) a_{1}} 2^{(j-1-n) m}\right\| u_{1} \|_{\mathcal{K}_{a_{1}+1,1}^{m+1}(U)} \\
& \leq C 2^{-(j-1) m} 2^{(j-1-n) m}\left\|u_{1}\right\|_{\mathcal{K}_{a_{1}+1,1}^{m+1}(U)}=C 2^{-n m}\left\|u_{1}\right\|_{\mathcal{K}_{a_{1}+1,1}^{m+1}(U)} .
\end{aligned}
$$

Then, we have proved the second estimate in this lemma. The first estimate can be proved similarly by Lemma 3.13 and the observation on the mesh size for the regions $G$ and $U$.

The following lemma gives the error bounds on the last patch $\mathbb{T}_{i, n}$ of triangles that have the vertex $Q_{i}$ as the common node.

Lemma 3.15. For $\kappa_{\beta}$ defined in (35), let $U \subset \mathcal{T}_{n}$ be the union of triangles that intersect $\mathbb{T}_{i, n}$. Suppose $\mathbb{T}_{i, 0} \subset \mathcal{V}$ near $Q_{i}$. Then,

$$
\begin{gathered}
\left\|u_{0}-\Pi u_{0}\right\|_{H_{+}^{1}\left(\mathbb{T}_{i, n}\right)} \leq C 2^{-n m}\left\|u_{0}\right\|_{\mathcal{K}_{a_{0}+1,1}^{m+1}(U)} \\
\left.\| u_{1}-\Pi u_{1}\right) \|_{H_{-}^{1}\left(\mathbb{T}_{i, n}\right)} \leq C 2^{-n m}\left(\left\|u_{1}\right\|_{\mathcal{K}_{a_{1}+1,1}^{m+1}(U)}+\left\|\vartheta^{-a_{1}} r^{-1} u_{1}\right\|_{L_{1}^{2}(U)}\right) .
\end{gathered}
$$

Proof. Definition 3.10 shows that the mesh on $U$ has the size $\mathcal{O}\left(\kappa_{\beta}^{n}\right)$. Let $\zeta$ be a smooth function, such that $\zeta=0$ in the small neighborhood of the vertex $Q_{i}$ and $\zeta=1$ on a region containing all the other nodes in $\mathbb{T}_{i, n}$. Let $v=u_{1}-\zeta u_{1}$. Note that $\Pi u_{1}=\Pi\left(\zeta u_{1}\right)$ and $\mathbb{T}_{i, 0}=\mathbb{T}_{i, n} / \lambda$, where $\lambda=\kappa_{\beta}^{n}$. If $Q_{i}$ is on the $z$-axis, by Lemma 3.12, we have

$$
\begin{gathered}
\left\|r^{-1} \zeta u_{1}\right\|_{L_{1}^{2}\left(\mathbb{T}_{i, n}\right)}=\lambda^{1 / 2}\left\|r_{\lambda}^{-1} \zeta_{\lambda}\left(u_{1}\right)_{\lambda}\right\|_{L_{1}^{2}\left(\mathbb{T}_{i, 0}\right)} \\
\leq C \lambda^{1 / 2}\left\|r_{\lambda}^{-1}\left(u_{1}\right)_{\lambda}\right\|_{L_{1}^{2}\left(\mathbb{T}_{i, 0}\right)}=C\left\|r^{-1} u_{1}\right\|_{L_{1}^{2}\left(\mathbb{T}_{i, n}\right)},
\end{gathered}
$$

and

$\left\|\zeta u_{1}\right\|_{\mathcal{K}_{1,1}^{m}\left(\mathbb{T}_{i, n}\right)}=\lambda^{1 / 2}\left\|\zeta_{\lambda}\left(u_{1}\right)_{\lambda}\right\|_{\mathcal{K}_{1,1}^{m}\left(\mathbb{T}_{i, 0}\right)} \leq C \lambda^{1 / 2}\left\|\left(u_{1}\right)_{\lambda}\right\|_{\mathcal{K}_{1,1}^{m}\left(\mathbb{T}_{i, 0}\right)}=C\left\|u_{1}\right\|_{\mathcal{K}_{1,1}^{m}\left(\mathbb{T}_{i, n}\right)}$.

Therefore, by Lemma 2.9, (10), Lemma 3.7, Lemma 3.12, and the estimates above,

$$
\begin{gathered}
\left\|r^{-1}\left(u_{1}-\Pi u_{1}\right)\right\|_{L_{1}^{2}\left(\mathbb{T}_{i, n}\right)}+\left\|u_{1}-\Pi u_{1}\right\|_{H_{1}^{1}\left(\mathbb{T}_{i, n}\right)} \\
\leq\left\|r^{-1} v\right\|_{L_{1}^{2}\left(\mathbb{T}_{i, n}\right)}+\left\|r^{-1}\left(\zeta u_{1}-\Pi u_{1}\right)\right\|_{L_{1}^{2}\left(\mathbb{T}_{i, n}\right)}+\|v\|_{H_{1}^{1}\left(\mathbb{T}_{i, n}\right)}+\left\|\zeta u_{1}-\Pi u_{1}\right\|_{\mathcal{K}_{1,1}^{1}\left(\mathbb{T}_{i, n}\right)} \\
\leq C\left(\left\|r^{-1} u_{1}\right\|_{L_{1}^{2}\left(\mathbb{T}_{i, n}\right)}+\left\|u_{1}\right\|_{\mathcal{K}_{1,1}^{1}\left(\mathbb{T}_{i, n}\right)}\right. \\
+\lambda^{1 / 2}\left(\left\|r_{\lambda}^{-1}\left(\zeta_{\lambda}\left(u_{1}\right)_{\lambda}-\Pi\left(u_{1}\right)_{\lambda}\right)\right\|_{L_{1}^{2}\left(\mathbb{T}_{i, 0}\right)}+\left\|\zeta_{\lambda}\left(u_{1}\right)_{\lambda}-\Pi\left(u_{1}\right)_{\lambda}\right\|_{\mathcal{K}_{1,1}^{1}\left(\mathbb{T}_{i, 0}\right)}\right) \\
\leq C\left(\left\|r^{-1} u_{1}\right\|_{L_{1}^{2}\left(\mathbb{T}_{i, n}\right)}+\left\|u_{1}\right\|_{\mathcal{K}_{1,1}^{1}\left(\mathbb{T}_{i, n}\right)}+\lambda^{1 / 2}\left|\zeta_{\lambda}\left(u_{1}\right)_{\lambda}\right|_{H_{1}^{m+1}\left(U_{\lambda}\right)}\right) \\
\leq C\left(\left\|r^{-1} u_{1}\right\|_{L_{1}^{2}\left(\mathbb{T}_{i, n}\right)}+\left\|u_{1}\right\|_{\mathcal{K}_{1,1}^{1}\left(\mathbb{T}_{i, n}\right)}+\lambda^{1 / 2}\left\|\left(u_{1}\right)_{\lambda}\right\|_{\mathcal{K}_{1,1}^{m+1}\left(U_{\lambda}\right)}\right) \\
=C\left(\left\|r^{-1} u_{1}\right\|_{L_{1}^{2}\left(\mathbb{T}_{i, n}\right)}+\left\|u_{1}\right\|_{\mathcal{K}_{1,1}^{m+1}(U)}\right) .
\end{gathered}
$$

If $Q_{i}$ is not on the $z$-axis, $r$ is bounded away from 0 , by Lemma 3.12,

$$
\begin{gathered}
\left\|r^{-1} \zeta u_{1}\right\|_{L_{1}^{2}\left(\mathbb{T}_{i, n}\right)}=\left\|\left(r^{-1} \vartheta\right) \vartheta^{-1} \zeta u_{1}\right\|_{L_{1}^{2}\left(\mathbb{T}_{i, n}\right)} \leq C\left\|\zeta u_{1}\right\|_{\mathcal{K}_{1,1}^{0}\left(\mathbb{T}_{i, n}\right)} \\
\leq C\left\|\zeta_{\lambda}\left(u_{1}\right)_{\lambda}\right\|_{\mathcal{K}_{1,1}^{0}\left(\mathbb{T}_{i, 0}\right)} \leq C\left\|\left(u_{1}\right)_{\lambda}\right\|_{\mathcal{K}_{1,1}^{0}\left(\mathbb{T}_{i, 0}\right)} \leq C\left\|u_{1}\right\|_{\mathcal{K}_{1,1}^{0}\left(\mathbb{T}_{i, n}\right)} ; \\
\left\|\zeta u_{1}\right\|_{\mathcal{K}_{1,1}^{m}\left(\mathbb{T}_{i, n}\right)} \leq C\left\|\zeta_{\lambda}\left(u_{1}\right)_{\lambda}\right\|_{\mathcal{K}_{1,1}^{m}\left(\mathbb{T}_{i, 0}\right)} \leq C\left\|\left(u_{1}\right)_{\lambda}\right\|_{\mathcal{K}_{1,1}^{m}\left(\mathbb{T}_{i, 0}\right)} \leq C\left\|u_{1}\right\|_{\mathcal{K}_{1,1}^{m}\left(\mathbb{T}_{i, n}\right)}
\end{gathered}
$$


Thus, by Lemma 2.8, Lemma 3.8, Lemma 3.12, and the estimates above,

$$
\begin{gathered}
\left\|r^{-1}\left(u_{1}-\Pi u_{1}\right)\right\|_{L_{1}^{2}\left(\mathbb{T}_{i, n}\right)}+\left\|u_{1}-\Pi u_{1}\right\|_{H_{1}^{1}\left(\mathbb{T}_{i, n}\right)} \\
\leq\left\|r^{-1} v\right\|_{L_{1}^{2}\left(\mathbb{T}_{i, n}\right)}+\left\|r^{-1}\left(\zeta u_{1}-\Pi u_{1}\right)\right\|_{L_{1}^{2}\left(\mathbb{T}_{i, n}\right)}+\|v\|_{H_{1}^{1}\left(\mathbb{T}_{i, n}\right)}+\left\|\zeta u_{1}-\Pi u_{1}\right\|_{H_{1}^{1}\left(\mathbb{T}_{i, n}\right)} \\
\leq C\left(\left\|u_{1}\right\|_{\mathcal{K}_{1,1}^{1}\left(\mathbb{T}_{i, n}\right)}+\left\|\zeta u_{1}-\Pi u_{1}\right\|_{\mathcal{K}_{1,1}^{1}\left(\mathbb{T}_{i, n}\right)}\right) \\
\leq C\left(\left\|u_{1}\right\|_{\mathcal{K}_{1,1}^{1}\left(\mathbb{T}_{i, n}\right)}+\left\|\zeta_{\lambda}\left(u_{1}\right)_{\lambda}-\Pi\left(u_{1}\right)_{\lambda}\right\|_{\mathcal{K}_{1,1}^{1}\left(\mathbb{T}_{i, 0}\right)}\right) \\
(40) \leq C\left(\left\|u_{1}\right\|_{\mathcal{K}_{1,1}^{1}\left(\mathbb{T}_{i, n}\right)}+\left\|\zeta_{\lambda}\left(u_{1}\right)_{\lambda}\right\|_{\mathcal{K}_{1,1}^{m+1}\left(\mathbb{T}_{i, 0}\right)}\right) \leq C\left\|u_{1}\right\|_{\mathcal{K}_{1,1}^{m+1}\left(\mathbb{T}_{i, n}\right)} .
\end{gathered}
$$

Note that $\max _{\mathbf{x} \in \mathbb{T}_{i, n}} \vartheta(\mathbf{x})=\mathcal{O}\left(\kappa_{\beta}^{n}\right)$. Combining (39) and (40), we have

$$
\begin{aligned}
& \left\|r^{-1}\left(u_{1}-\Pi u_{1}\right)\right\|_{L_{1}^{2}\left(\mathbb{T}_{i, n}\right)}+\left\|u_{1}-\Pi u_{1}\right\|_{H_{1}^{1}\left(\mathbb{T}_{i, n}\right)} \leq C\left(\left\|r^{-1} u_{1}\right\|_{L_{1}^{2}\left(\mathbb{T}_{i, n}\right)}+\left\|u_{1}\right\|_{\mathcal{K}_{1,1}^{m+1}(U)}\right) \\
& \leq C \kappa_{1}^{n a_{1}}\left(\left\|\vartheta^{-a_{1}} r^{-1} u_{1}\right\|_{L_{1}^{2}\left(\mathbb{T}_{i, n}\right)}+\left\|u_{1}\right\|_{\mathcal{K}_{a_{1}+1,1}^{m+1}(U)}\right) \\
& \leq C 2^{-n m}\left(\left\|\vartheta^{-a_{1}} r^{-1} u_{1}\right\|_{L_{1}^{2}\left(\mathbb{T}_{i, n}\right)}+\left\|u_{1}\right\|_{\mathcal{K}_{a_{1}+1,1}^{m+1}(U)}\right) .
\end{aligned}
$$

This completes the proof of the second estimate of the lemma. The first estimate follows from a similar process using Lemmas 3.12, 2.8, 2.9, 3.7, and 3.8.

The local estimates above lead to the main result on the convergence rate of our finite element method.

Theorem 3.16. Let $u_{\beta}$ be the solution of equation (1) and $u_{n}^{\beta} \in \mathbb{V}_{n}^{\beta}$ be the finite element solution defined in (30). Recall $\kappa_{\beta}$ from (35) for $0<a_{\beta}<\Upsilon_{\beta}$ as defined in Theorem 3.5. Then, for $f_{0} \in \mathcal{K}_{a_{0}-1,+}^{m-1}(\Omega)$ and $f_{1} \in \mathcal{K}_{a_{1}-1,-}^{m-1}(\Omega)$,

$$
\begin{gathered}
\left\|u_{0}-u_{n}^{0}\right\|_{H_{+}^{1}(\Omega)} \leq C \operatorname{dim}\left(\mathbb{V}_{n}^{0}\right)^{-m / 2}\left\|f_{0}\right\|_{\mathcal{K}_{a_{0}-1,+}^{m-1}(\Omega)}, \\
\left\|u_{1}-u_{n}^{1}\right\|_{H_{-}^{1}(\Omega)} \leq C \operatorname{dim}\left(\mathbb{V}_{n}^{1}\right)^{-m / 2}\left\|f_{1}\right\|_{\mathcal{K}_{a_{1}-1,-}^{m-1}(\Omega)} .
\end{gathered}
$$

where $\operatorname{dim}\left(\mathbb{V}_{n}^{\beta}\right)=\mathcal{O}\left(4^{n}\right)$ is the dimension of $\mathbb{V}_{n}^{\beta}$ and $m \geq 1$.

Proof. The proof follows by summing up the estimates in Lemmas 3.11, 3.14, and 3.15, together with the regularity results in Theorem 3.5.

Remark 3.17. The graded meshes from the $\kappa$-refinements are nested and consist of shape-regular triangles. Various numerical tests for the linear finite element methods $(m=1)$ approximating singular solutions in the case $\beta=0$ can be found in [23], which convincingly verify Theorem 3.16. As mentioned in Remark 3.6, instead of a uniform $\alpha_{\beta}$ for all vertices, we can choose different regularity indices $a_{\beta}$ near different vertices $Q_{i}$. This will result in different grading parameters $\kappa_{\beta}$ close to the vertices. Nevertheless, as long as $0<a_{\beta}<\eta_{\beta}^{i}$ for each $Q_{i}$, we shall have the optimal convergence rate indicated in Theorem 3.16. This flexibility can help to improve the shape regularity of the triangulation.

\section{The MUltigrid ALGORITHM}

From now on, we concentrate on the multigrid analysis for the linear finite element approximation of equation (1) on graded meshes given in the last section. 
4.1. The multigrid V-cycle algorithm. We denote the $\beta$-dependent linear finite element spaces

$$
\mathbb{V}_{0}^{\beta} \subset \mathbb{V}_{1}^{\beta} \subset \cdots \subset \mathbb{V}_{K}^{\beta}
$$

that are defined on graded meshes $\mathcal{T}_{k}$ in the last section, $0 \leq k \leq K$, where the parameter $\kappa_{\beta}$ is chosen as in (35) for $m=1$. For simplicity, we assume that there is only one singular vertex $Q$ for the solution. Namely, the solution is in $H_{1}^{2}$ (or $\eta_{\beta}^{i}>1$ ) except in the neighborhood of $Q$. Therefore, the graded mesh $\left(\kappa_{\beta}<0.5\right)$ is implemented only for triangles touching $Q$, while the usual quasiuniform $(\kappa=0.5)$ decomposition is performed for other triangles. If we modify the weight function in the definition of the space $\mathcal{K}_{\mu, 1}^{m}(\Omega)$, letting $\vartheta$ be the distance to $Q$ in its neighborhood $\mathcal{V}$ and be 1 in the neighborhoods of other vertices, then Theorem 3.16 in this case reads

$$
\begin{aligned}
\left\|u_{0}-u_{k}^{0}\right\|_{H_{+}^{1}(\Omega)} & \leq C \operatorname{dim}\left(\mathbb{V}_{k}^{0}\right)^{-1 / 2}\left\|f_{0}\right\|_{\mathcal{K}_{a_{0}-1,+}^{0}(\Omega)}, \\
\left\|u_{1}-u_{k}^{1}\right\|_{H_{-}^{1}(\Omega)} & \leq C \operatorname{dim}\left(\mathbb{V}_{k}^{1}\right)^{-1 / 2}\left\|f_{1}\right\|_{\mathcal{K}_{a_{1}-1}^{0}(\Omega)} .
\end{aligned}
$$

In this section, we use this modified function $\vartheta$ and also define $\nabla:=\left(\partial_{r}, \partial_{z}\right)^{t}$.

Let $\mathbb{T}_{k} \subset \mathcal{T}_{k}$ be the union of all the triangles in $\mathcal{T}_{k}$ touching $Q$. Define the $j$ th layer of $\mathcal{T}_{k}$,

$$
L_{j}:=\mathbb{T}_{j-1} \backslash \mathbb{T}_{j}, \quad 0 \leq j \leq k,
$$

where $\mathbb{T}_{-1}:=\mathcal{T}_{0}$. Then, we define the piecewise-constant function on $\mathcal{T}_{k}$

$$
\left.\omega_{k}\right|_{L_{j}}:=\left(2 \kappa_{\beta}\right)^{-j},
$$

where $\kappa_{\beta}=2^{-1 / a_{\beta}}<0.5$ is the grading parameter for $Q$.

Then, we define the following mesh-dependent weighted inner product

$$
\left(v_{k}, w_{k}\right)_{k}:=\int_{\Omega} \omega_{k}^{2} v_{k} w_{k} r d r d z, \quad \forall v_{k}, w_{k} \in \mathbb{V}_{k}^{\beta},
$$

and the norm induced by the inner product,

$$
\left\|v_{k}\right\|_{k}^{2}:=\int_{\Omega} \omega_{k}^{2} v_{k}^{2} r d r d z, \quad \forall v_{k} \in \mathbb{V}_{k}^{\beta} .
$$

Let $\mathcal{I}_{k-1}^{k}: \mathbb{V}_{k-1}^{\beta} \rightarrow \mathbb{V}_{k}^{\beta}$ be the coarse-to-fine operator, which is the natural injection. The fine-to-coarse operator $\mathcal{I}_{k}^{k-1}: \mathbb{V}_{k}^{\beta} \rightarrow \mathbb{V}_{k-1}^{\beta}$ is the transpose with respect to the inner product in (45),

$$
\left(\mathcal{I}_{k}^{k-1} v_{k}, w_{k-1}\right)_{k-1}:=\left(v_{k}, \mathcal{I}_{k-1}^{k} w_{k-1}\right)_{k}, \quad \forall v_{k} \in \mathbb{V}_{k}^{\beta}, \forall w_{k-1} \in \mathbb{V}_{k-1}^{\beta} .
$$

Let $A_{k}^{\beta}: \mathbb{V}_{k}^{\beta} \rightarrow \mathbb{V}_{k}^{\beta}$ be the operator associated with equation (30),

$$
\left(A_{k}^{\beta} v_{k}, w_{k}\right)_{k}:=a_{\beta}\left(v_{k}, w_{k}\right), \quad \forall v_{k}, w_{k} \in \mathbb{V}_{k}^{\beta} .
$$

In addition, let $f_{k}^{\beta} \in \mathbb{V}_{k}^{\beta}$ be the function, such that

$$
\left(f_{k}^{\beta}, v_{k}\right)_{k}=\left(f_{\beta}, v_{k}\right)_{L_{1}^{2}(\Omega)}, \quad \forall v_{k} \in \mathbb{V}_{k}^{\beta} .
$$

Thus, we are ready to formulate the abstract multigrid V-cycle algorithm for equation (1) with the Richardson smoother. 
Algorithm 4.1. (The V-cycle algorithm). The $k$ th level V-cycle algorithm produces $M G\left(k, f_{k}^{\beta}, z_{0}, l\right)$ as an approximate solution for

$$
A_{k}^{\beta} u_{k}^{\beta}=f_{k}^{\beta}
$$

with initial guess $z_{0} \in \mathbb{V}_{k}^{\beta}$, where $l$ denotes the number of pre-smoothing and postsmoothing steps.

For $k=0$, we define

$$
M G\left(0, f_{0}^{\beta}, z_{0}, l\right)=\left(A_{0}^{\beta}\right)^{-1} f_{0}^{\beta} .
$$

For $k \geq 1$, the approximate solution $M G\left(k, f_{k}^{\beta}, z_{0}, l\right)$ is computed recursively in three steps:

Pre-smoothing. For $1 \leq j \leq l$, compute $z_{j}$ by

$$
z_{j}=z_{j-1}+\gamma_{k}\left(f_{k}^{\beta}-A_{k}^{\beta} z_{j-1}\right)
$$

where the constant $\gamma_{k}$ is the damping factor that we will specify later.

Coarse grid correction. Let $g_{k-1}=\mathcal{I}_{k}^{k-1}\left(f_{k}^{\beta}-A_{k}^{\beta} z_{l}\right)$ be the restriction of the residue on the $k-1$ st level. Define $q \in \mathbb{V}_{k-1}^{\beta}$ by

$$
q:=M G\left(k-1, g_{k-1}, 0, l\right) .
$$

Then, we compute $z_{l+1}$ by

$$
z_{l+1}=z_{l}+\mathcal{I}_{k-1}^{k} q .
$$

Post-smoothing. For $l+2 \leq j \leq 2 l+1$, compute $z_{j}$ by

$$
z_{j}=z_{j-1}+\gamma_{k}\left(f_{k}^{\beta}-A_{k}^{\beta} z_{j-1}\right) .
$$

We then define $M G\left(k, f_{k}^{\beta}, z_{0}, l\right)=z_{2 l+1}$.

Remark 4.2. Let $E_{k}: \mathbb{V}_{k}^{\beta} \rightarrow \mathbb{V}_{k}^{\beta}$ be the error-propagation operator for the $k$ th level V-cycle algorithm defined above. Namely, $E_{k}\left(u_{k}^{\beta}-z_{0}\right)=u_{k}^{\beta}-M G\left(k, f_{k}^{\beta}, z_{0}, l\right)$. The relation below follows from a straightforward calculation:

$$
E_{k}=R_{k}^{l}\left(I d_{k}-\mathcal{I}_{k-1}^{k} P_{k}^{k-1}+\mathcal{I}_{k-1}^{k} E_{k-1} P_{k}^{k-1}\right) R_{k}^{l},
$$

where $I d_{k}$ is the identity operator on $\mathbb{V}_{k}^{\beta}, R_{k}: \mathbb{V}_{k}^{\beta} \rightarrow \mathbb{V}_{k}^{\beta}$ measuring the effect of the smoothing step is defined by

$$
R_{k}:=I d_{k}-\gamma_{k} A_{k}^{\beta},
$$

and $P_{k}^{k-1}: \mathbb{V}_{k}^{\beta} \rightarrow \mathbb{V}_{k-1}^{\beta}$ is the transpose of $\mathcal{I}_{k-1}^{k}$ with respect to the bilinear forms, i.e., for any $v_{k} \in \mathbb{V}_{k}^{\beta}$,

$$
a_{\beta}\left(P_{k}^{k-1} v_{k}, w_{k-1}\right)=a_{\beta}\left(v_{k}, \mathcal{I}_{k-1}^{k} w_{k-1}\right), \quad \forall w_{k} \in \mathbb{V}_{k-1}^{\beta} .
$$

Note that $P_{k}^{k-1}$ is in fact the projection on $\mathbb{V}_{k-1}^{\beta}$ with respect to $a_{\beta}(\cdot, \cdot)$. Then, for any $v_{k-1}, w_{k-1} \in \mathbb{V}_{k-1}^{\beta}$, we have

$$
a_{\beta}\left(P_{k}^{k-1} \mathcal{I}_{k-1}^{k} v_{k-1}, w_{k-1}\right)=a_{\beta}\left(\mathcal{I}_{k-1}^{k} v_{k-1}, \mathcal{I}_{k-1}^{k} w_{k-1}\right)=a_{\beta}\left(v_{k-1}, w_{k-1}\right) .
$$

Then, for any $v_{k} \in \mathbb{V}_{k-1}^{\beta}, w_{k} \in \mathbb{V}_{k}^{\beta}$,

$$
\begin{aligned}
& a_{\beta}\left(\mathcal{I}_{k-1}^{k} v_{k-1},\left(I d_{k}-\mathcal{I}_{k-1}^{k} P_{k}^{k-1}\right) w_{k}\right) \\
& =a_{\beta}\left(\mathcal{I}_{k-1}^{k} v_{k-1}, w_{k}\right)-a_{\beta}\left(\mathcal{I}_{k-1}^{k} v_{k-1}, \mathcal{I}_{k-1}^{k} P_{k}^{k-1} w_{k}\right) \\
& \left.=a_{\beta}\left(v_{k-1}, P_{k}^{k-1} w_{k}\right)-a\left(v_{k-1}, P_{k}^{k-1} \mathcal{I}_{k-1}^{k} P_{k}^{k-1} w_{k}\right)\right)=0 .
\end{aligned}
$$




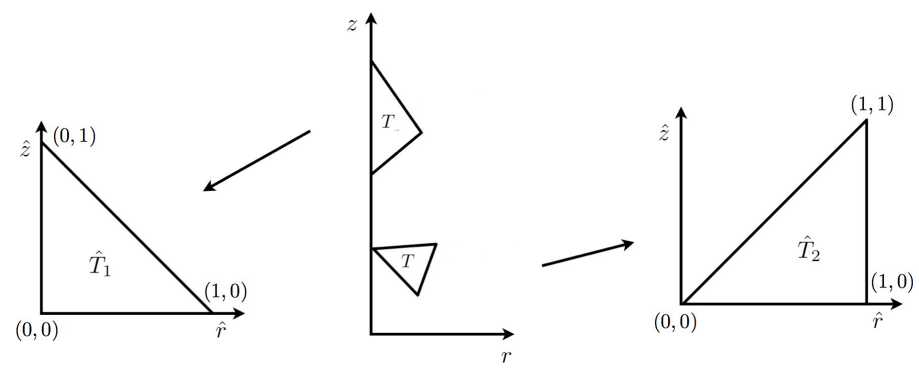

Figure 2. Reference triangles $\hat{T}_{1}$ and $\hat{T}_{2}$ corresponding to different triangles touching the $z$-axis.

For the sake of economy of notation, we use the same notation for the operators $I d_{k}, \mathcal{I}_{k-1}^{k}, \mathcal{I}_{k}^{k-1}, E_{k}, R_{k}$, and $P_{k}^{k-1}$, even though $\mathbb{V}_{k}^{0}$ and $\mathbb{V}_{k}^{1}$ are different subspaces of $H_{1}^{1}(\Omega)$ (see $\left.(29)\right)$.

Let $\left\{\mathbf{x}_{i}\right\}$ be the set of nodes in the triangulation $\mathcal{T}_{k}$ and $d_{i, k}$ be the diameter of the support of the basis function associated with the node $\mathbf{x}_{i}$. Throughout the text, by $A \simeq B$, we mean there are generic constants $C_{1}, C_{2}>0$, such that $C_{1} B \leq A \leq C_{2} B$. For a triangle $T, r_{\max }(T):=\max _{\mathbf{x} \in T} r(\mathbf{x})$ and $r_{\min }(T):=\min _{\mathbf{x} \in T} r(\mathbf{x})$. For a triangle $T \in \mathcal{T}_{k}$ away from the $z$-axis, we denote by $\hat{T}$ its standard reference triangle of diameter one. If $T$ touches the $z$-axis, its reference triangle $\hat{T}$ will be either $\hat{T}_{1}$ or $\hat{T}_{2}$ (Figure 2), to preserve the intersection set between $T$ and the $z$-axis. For $v_{k} \in \mathbb{V}_{k}^{\beta}$ and $\mathbf{x} \in T$, we define $\hat{v}_{k}(\hat{\mathbf{x}}):=v_{k}(\mathbf{x})$, where $\hat{\mathbf{x}} \in \hat{T}$ is the image of $\mathbf{x}$ after the affine mapping.

We now investigate necessary properties of the inner products and of the operators in Algorithm 4.1.

Lemma 4.3. Let $T_{i} \in \mathcal{T}_{k}$ be a triangle and $h_{i, k}$ be its diameter. Then,

$$
\int_{T_{i}} v_{k}^{2} r d r d z \simeq h_{i, k}^{2} \sum_{\mathbf{x}_{l} \in T_{i}} v_{k}^{2}\left(\mathbf{x}_{l}\right) \max \left(d_{l, k}, r\left(\mathbf{x}_{l}\right)\right), \quad \forall v_{k} \in \mathbb{V}_{k}^{\beta},
$$

where $\mathbf{x}_{l}, 1 \leq l \leq 3$, is the vertex of $T_{i}$ and $d_{l, k}$ is defined as above.

Proof. Case I: $T_{i} \cap\{r=0\}=\emptyset$. Thus, $r_{\max }\left(T_{i}\right) / r_{\min }\left(T_{i}\right)<M$ with a uniform constant $M>0$ for any $T_{i}$. We map $T_{i}$ to the reference triangle $\hat{T}$. Based on the norm equivalence on finite dimensional spaces, we have

$$
\begin{aligned}
\int_{T_{i}} v_{k}^{2} r d r d z & \simeq r_{\max }\left(T_{i}\right) \int_{T_{i}} v_{k}^{2} d r d z=r_{\max }\left(T_{i}\right)\left|T_{i}\right| \int_{\hat{T}} \hat{v}_{k}^{2} d \hat{r} d \hat{z} \\
& \simeq r_{\max }\left(T_{i}\right) h_{i, k}^{2} \sum_{\hat{\mathbf{x}}_{l} \in \hat{T}} \hat{v}_{k}^{2}\left(\hat{\mathbf{x}}_{l}\right) \simeq h_{i, k}^{2} \sum_{\mathbf{x}_{l} \in T_{i}} v_{k}^{2}\left(x_{l}\right) \max \left(d_{l, k}, r\left(\mathbf{x}_{l}\right)\right),
\end{aligned}
$$

where we used the fact that $r_{\max }\left(T_{i}\right) \simeq \max \left(d_{l, k}, r\left(\mathbf{x}_{l}\right)\right)$ for any triangle away from the $z$-axis.

Case II: $T_{i} \cap\{r=0\} \neq \emptyset$. Let $\left\{\lambda_{m}\right\}_{m=1,2,3}$ be the barycentric coordinates associated with the three vertices of $T_{i}$.

1. $\left(T_{i} \cap\{r=0\}\right.$ is a line segment.) We map $T_{i}$ to the the first reference $\hat{T}_{1}$. Without loss of generality, we assume the first node of $T_{i}$ is away from the $z$-axis. 
Let $r_{1}$ be the distance from its first node to the $z$-axis. Note that $r_{1} \simeq h_{i, k}$. Based on the norm equivalence on finite dimensional spaces, we have

$$
\begin{aligned}
\int_{T_{i}} v_{k}^{2} r d r d z & =r_{1} \int_{T_{i}} v_{k}^{2} \lambda_{1} d r d z=r_{1}\left|T_{i}\right| \int_{\hat{T}_{1}} \hat{v}_{k}^{2} \hat{\lambda}_{1} d \hat{r} d \hat{z} \\
& \simeq h_{i, k}^{3} \sum_{\hat{\mathbf{x}}_{l} \in \hat{T}_{1}} \hat{v}_{k}^{2}\left(\hat{\mathbf{x}}_{l}\right) \simeq h_{i, k}^{2} \sum_{\mathbf{x}_{l} \in T_{i}} v_{k}^{2}\left(\mathbf{x}_{l}\right) \max \left(d_{l, k}, r\left(\mathbf{x}_{l}\right)\right) .
\end{aligned}
$$

2. $\left(T_{i} \cap\{r=0\}\right.$ is a point.) We map $T_{i}$ to the second reference $\hat{T}_{2}$. Suppose that its third vertex is on the $z$-axis. Denote by $r_{1}$ and $r_{2}$ the distances form the first and second vertices of $T_{i}$ to the $z$-axis, respectively. Then, for any $\mathbf{x} \in T_{i}$, $r(\mathbf{x})=r_{1} \lambda_{1}(\mathbf{x})+r_{2} \lambda_{2}(\mathbf{x})$ with $r_{1} \simeq r_{2} \simeq h_{i, k}$. Using the norm equivalence on finite element spaces, we have

$$
\begin{aligned}
\int_{T_{i}} v_{k}^{2} r d r d z & =\int_{T_{i}} v_{k}^{2}\left(r_{1} \lambda_{1}+r_{2} \lambda_{2}\right) d r d z \simeq h_{i, k}\left|T_{i}\right| \int_{\hat{T}_{2}} \hat{v}_{k}^{2}\left(\hat{\lambda}_{1}+\hat{\lambda}_{2}\right) d \hat{r} d \hat{z} \\
& \simeq h_{i, k}^{3} \sum_{\hat{\mathbf{x}}_{l} \in \hat{T}_{2}} \hat{v}_{k}^{2}\left(\hat{\mathbf{x}}_{l}\right) \simeq h_{i, k}^{2} \sum_{\mathbf{x}_{l} \in T_{i}} v_{k}^{2}\left(\mathbf{x}_{l}\right) \max \left(d_{l, k}, r\left(\mathbf{x}_{l}\right)\right) .
\end{aligned}
$$

Hence, the lemma is proved for any $T_{i} \in \mathcal{T}_{k}$.

Lemma 4.4. The weighted inner product in (45) satisfies

$$
\left(v_{k}, v_{k}\right)_{k} \simeq \operatorname{dim}\left(\mathbb{V}_{k}^{\beta}\right)^{-1} \sum_{i} v_{k}^{2}\left(\mathbf{x}_{i}\right) \max \left(d_{i, k}, r\left(\mathbf{x}_{i}\right)\right), \quad \forall v_{k} \in \mathbb{V}_{k}^{\beta} .
$$

Proof. Recall $\operatorname{dim}\left(\mathbb{V}_{k}^{\beta}\right) \simeq 4^{k}$. Thus, for any $v_{k} \in \mathbb{V}_{k}^{\beta}$, we have

$$
\begin{aligned}
& \operatorname{dim}\left(\mathbb{V}_{k}^{\beta}\right)^{-1} \sum_{i=1}^{\operatorname{dim}\left(\mathbb{V}_{k}^{\beta}\right)} v_{k}^{2}\left(\mathbf{x}_{i}\right) \max \left(d_{i, k}, r\left(\mathbf{x}_{i}\right)\right) \\
& \simeq \sum_{i=1}^{\operatorname{dim}\left(\mathbb{V}_{k}^{\beta}\right)}(2 \kappa)^{-2 \Im(i)}\left(2^{2(\Im(i)-k)} \kappa^{2 \Im(i)}\right) v_{k}^{2}\left(\mathbf{x}_{i}\right) \max \left(d_{i, k}, r\left(\mathbf{x}_{i}\right)\right),
\end{aligned}
$$

where the function $\Im: \mathbb{N} \rightarrow \mathbb{N} \cup\{0\}$ is such that $\Im(i)=j$ if the node $\mathbf{x}_{i}$ is in the $j$ th layer $L_{j}$ of $\mathcal{T}_{k}$. By Definition 3.10, for a node $\mathbf{x}_{i} \in L_{j}$, the diameter of a triangle $T_{l}$ touching $\mathbf{x}_{i}$ satisfies $h_{l, k} \simeq 2^{j-k} \kappa^{j}$. Therefore, using Lemma 4.3, we have

$$
\begin{aligned}
& \operatorname{dim}\left(\mathbb{V}_{k}^{\beta}\right)^{-1} \sum_{i=1}^{\operatorname{dim}\left(\mathbb{V}_{k}^{\beta}\right)} v_{k}^{2}\left(\mathbf{x}_{i}\right) \max \left(d_{i, k}, r\left(\mathbf{x}_{i}\right)\right) \\
& \simeq \sum_{T_{l} \in \mathcal{T}_{k}} \sum_{\mathbf{x}_{i} \in T_{l}}(2 \kappa)^{-2 \Im(i)}\left(2^{2(\Im(i)-k)} \kappa^{2 \Im(i)}\right) v_{k}^{2}\left(\mathbf{x}_{i}\right) \max \left(d_{i, k}, r\left(\mathbf{x}_{i}\right)\right) \\
& \left.\simeq \sum_{T_{l} \in \mathcal{T}_{k}} \omega_{k}^{2}\right|_{T_{l}} h_{l, k}^{2} \sum_{\mathbf{x}_{i} \in T_{l}} v_{k}^{2}\left(\mathbf{x}_{i}\right) \max \left(d_{i, k}, r\left(\mathbf{x}_{i}\right)\right) \\
& \left.\simeq \sum_{T_{l} \in \mathcal{T}_{k}} \omega_{k}^{2}\right|_{T_{l}} \int_{T_{l}} v_{k}^{2} r d r d z=\int_{\Omega} \omega_{k}^{2} v_{k}^{2} r d r d z=\left(v_{k}, v_{k}\right)_{k},
\end{aligned}
$$

which completes the proof. 
Lemma 4.5. The spectral radus of $A_{k}^{\beta}$ defined in (46) satisfies

$$
0<\rho\left(A_{k}^{\beta}\right) \leq C \operatorname{dim}\left(\mathbb{V}_{k}^{\beta}\right) .
$$

Proof. According to the definition, $A_{k}^{\beta}$ is symmetric positive definite (SPD) with respect to the inner product $(\cdot, \cdot)_{k}$. Therefore, it has a discrete spectrum and all its eigenvalues are real and positive. Thus, we only need to show for any $\mathbb{V}_{k}^{\beta} \ni v_{k} \neq 0$,

$$
\frac{\left(A_{k}^{\beta} v_{k}, v_{k}\right)_{k}}{\left(v_{k}, v_{k}\right)_{k}} \leq C \operatorname{dim}\left(\mathbb{V}_{k}^{\beta}\right)
$$

Recall that near the singular vertex $Q$, the grading parameter $\kappa_{\beta}=2^{-1 / a_{\beta}}<0.5$. If a triangle $T_{i}$ is in the $j$ th layer $L_{j}$ of $\mathcal{T}_{k}, 0 \leq j \leq k$, its diameter $h_{i, k} \simeq \kappa^{j} 2^{j-k}$, and so is the diameter $d_{l, k}$ of the support of the basis function associated with the vertex $\mathbf{x}_{l}$ of $T_{i}$.

Case I: $T_{i} \cap\{r=0\}=\emptyset$. Recall that $r_{\max }\left(T_{i}\right) / r_{\min }\left(T_{i}\right) \leq M$ is bounded with $M>0$ for any $T_{i}$. Then,

$$
\begin{aligned}
\int_{T_{i}}\left(\nabla v_{k} \cdot \nabla v_{k}\right) r d r d z & \simeq r_{\max }\left(T_{i}\right) \int_{T_{i}} \nabla v_{k} \cdot \nabla v_{k} d r d z \leq C h_{i, k}^{-2} r_{\max }\left(T_{i}\right) \int_{T_{i}} v_{k}^{2} d r d z \\
(52) & \simeq r_{\max }\left(T_{i}\right) \sum_{\mathbf{x}_{l} \in T_{i}} v_{k}^{2}\left(\mathbf{x}_{l}\right) \simeq \sum_{\mathbf{x}_{l} \in T_{i}} v_{k}^{2}\left(\mathbf{x}_{l}\right) r\left(\mathbf{x}_{l}\right),
\end{aligned}
$$

where we used the usual $H^{1}-L^{2}$ inverse inequality in the finite element space and the norm equivalence on finite dimensional spaces.

Note $r_{\max }\left(T_{i}\right)^{-1} \leq C h_{i, k}^{-1}$. By the norm equivalence on finite dimensional spaces,

$$
\begin{aligned}
\int_{T_{i}} r^{-1} v_{k}^{2} d r d z & \simeq r_{\max }\left(T_{i}\right)^{-1} \int_{T_{i}} v_{k}^{2} d r d z=r_{\max }\left(T_{i}\right)^{-1}\left|T_{i}\right| \int_{\hat{T}} \hat{v}_{k}^{2} d \hat{r} d \hat{z} \\
& \leq C h_{i, k} \sum_{\hat{\mathbf{x}}_{l} \in \hat{T}_{k}} \hat{v}_{k}^{2}\left(\hat{\mathbf{x}}_{l}\right) \leq C \sum_{\mathbf{x}_{l} \in T_{i}} v_{k}^{2}\left(\mathbf{x}_{l}\right) \max \left(d_{l, k}, r\left(\mathbf{x}_{l}\right)\right) .
\end{aligned}
$$

Case II: $T_{i} \cap\{r=0\} \neq \emptyset$. Let $\left\{\lambda_{m}\right\}_{m=1,2,3}$ be the barycentric coordinates associated with the three vertices of $T_{i}$.

1. ( $T_{i} \cap\{r=0\}$ is a line segment.) We map $T_{i}$ to $\hat{T}_{1}$. Suppose the first node of $T_{i}$ is away from the $z$-axis. Let $r_{1}$ be the distance from the first node to the $z$-axis. Then, $r_{1} \simeq h_{i, k}$. Based on the norm equivalence on finite dimensional spaces,

$$
\int_{\hat{T}_{1}}\left(\nabla \hat{v}_{k} \cdot \nabla \hat{v}_{k}\right) \hat{\lambda}_{1} d \hat{r} d \hat{z}+\int_{\hat{T}_{1}} \hat{v}_{k}^{2} \hat{\lambda}_{1} d \hat{r} d \hat{z} \leq C \int_{\hat{T}_{1}} \hat{v}_{k}^{2} d \hat{r} d \hat{z}
$$

since each side above defines the square of a norm for $\hat{v}_{k}$. Then,

$$
\begin{aligned}
\int_{T_{i}}\left(\nabla v_{k} \cdot \nabla v_{k}\right) r d r d z & =r_{1} \int_{T_{i}}\left(\nabla v_{k} \cdot \nabla v_{k}\right) \lambda_{1} d r d z \\
& \simeq r_{1}\left|T_{i}\right| \int_{\hat{T}_{1}} h_{i, k}^{-2}\left(\nabla \hat{v}_{k} \cdot \nabla \hat{v}_{k}\right) \hat{\lambda}_{1} d \hat{r} d \hat{z} \leq C r_{1} \int_{\hat{T}_{1}} \hat{v}_{k}^{2} d \hat{r} d \hat{z} \\
& \simeq r_{1} \sum_{\hat{\mathbf{x}}_{l} \in \hat{T}_{1}} \hat{v}_{k}^{2}\left(\hat{\mathbf{x}}_{l}\right) \simeq \sum_{\mathbf{x}_{l} \in T_{i}} v_{k}^{2}\left(\mathbf{x}_{l}\right) \max \left(d_{l, k}, r\left(\mathbf{x}_{l}\right)\right),
\end{aligned}
$$

where we used the scaling argument, the norm equivalence on finite dimensional spaces, and (54). 
For $v_{k} \in \mathbb{V}_{k}^{1},\left.v_{k}\right|_{T_{i}}$ is a linear function that vanishes on the $z$-axis. Therefore, $\left.v_{k}\right|_{T_{i}}=\nu_{k} r$ for a constant $\nu_{k}$. Then, we have

$$
\begin{aligned}
\int_{T_{i}} r^{-1} v_{k}^{2} d r d z & =\int_{T_{i}} \nu_{k}^{2} r d r d z=r_{1}\left|T_{i}\right| \int_{\hat{T}_{1}} \nu_{k}^{2} \hat{\lambda}_{1} d \hat{r} d \hat{z} \simeq h_{i, k}^{3} \nu_{k}^{2} \\
& \simeq h_{i, k} \sum_{\mathbf{x}_{l} \in T_{i}} v_{k}^{2}\left(\mathbf{x}_{l}\right) \simeq \sum_{\mathbf{x}_{l} \in T_{i}} v_{k}^{2}\left(\mathbf{x}_{l}\right) \max \left(d_{l, k}, r\left(\mathbf{x}_{l}\right)\right)
\end{aligned}
$$

where we used the norm equivalence on finite dimensional spaces.

2. $\left(T_{i} \cap\{r=0\}\right.$ is a point.) We map $T_{i}$ to $\hat{T}_{2}$. Suppose that its first two vertices are not on the $z$-axis. Denote by $r_{1}$ and $r_{2}$ the distances from the first vertex and the second vertex of $T_{i}$ to the $z$-axis, respectively. For any point $\mathbf{x} \in T_{i}$, $r(\mathbf{x})=r_{1} \lambda_{1}(\mathbf{x})+r_{2} \lambda_{2}(\mathbf{x})$ and $r_{1} \simeq r_{2} \simeq h_{i, k}$. Using the norm equivalence on finite dimensional spaces, we have

$$
\int_{\hat{T}_{2}}\left(\nabla \hat{v}_{k} \cdot \nabla \hat{v}_{k}\right)\left(\hat{\lambda}_{1}+\hat{\lambda}_{2}\right) d \hat{r} d \hat{z}+\int_{\hat{T}_{2}} \hat{v}_{k}^{2}\left(\hat{\lambda}_{1}+\hat{\lambda}_{2}\right) d \hat{r} d \hat{z} \leq C \int_{\hat{T}_{2}} \hat{v}_{k}^{2} d \hat{r} d \hat{z}
$$

since each side of (57) defines the square of a norm for $\hat{v}_{k}$. Similarly, we have

$$
\begin{aligned}
& \int_{T_{i}}\left(\nabla v_{k} \cdot \nabla v_{k}\right) r d r d z \simeq h_{i, k}\left|T_{i}\right| \int_{\hat{T}_{2}} h_{i, k}^{-2}\left(\nabla \hat{v}_{k} \cdot \nabla \hat{v}_{k}\right)\left(\hat{\lambda}_{1}+\hat{\lambda}_{2}\right) d \hat{r} d \hat{z} \\
& \leq C h_{i, k} \int_{\hat{T}_{2}} \hat{v}_{k}^{2} d \hat{r} d \hat{z} \simeq h_{i, k} \sum_{\hat{\mathbf{x}}_{l} \in \hat{T}_{2}} \hat{v}_{k}^{2}\left(\hat{\mathbf{x}}_{l}\right) \simeq \sum_{x_{l} \in T_{i}} v_{k}^{2}\left(\mathbf{x}_{l}\right) \max \left(d_{l, k}, r\left(\mathbf{x}_{l}\right)\right) .
\end{aligned}
$$

For $v_{k} \in \mathbb{V}_{k}^{1}$, we can write the linear function $\left.v_{k}\right|_{T_{i}}=\sum_{l} \nu_{l} \phi_{l}$, where for $l=1,2$, $\nu_{l}$ is a constant and $\phi_{l}$ is the linear basis function associated with the $l$ th vertex of $T_{i}$. It was shown that $\int_{T_{i}} r^{-1} \phi_{l}^{2} d r d z<\infty$ in [21]. Therefore, $\int_{T_{i}} r^{-1} v_{k}^{2} d r d z$ is bounded. We then have

$$
\begin{aligned}
& \int_{T_{i}} r^{-1} v_{k}^{2} d r d z=\int_{T_{i}}\left(r_{1} \lambda_{1}+r_{2} \lambda_{2}\right)^{-1} v_{k}^{2} d r d z \simeq h_{i, k}^{-1}\left|T_{i}\right| \int_{\hat{T}_{2}}\left(\hat{\lambda}_{1}+\hat{\lambda}_{2}\right)^{-1} \hat{v}_{k}^{2} d \hat{r} d \hat{z} \\
& (59) \quad \simeq h_{i, k} \sum_{\hat{\mathbf{x}}_{l} \in \hat{T}_{2}} \hat{v}_{k}^{2}\left(\hat{\mathbf{x}}_{l}\right)=h_{i, k} \sum_{\mathbf{x}_{l} \in T_{i}} v_{k}^{2}\left(\mathbf{x}_{l}\right) \simeq \sum_{\mathbf{x}_{l} \in T_{i}} v_{k}^{2}\left(\mathbf{x}_{l}\right) \max \left(d_{l, k}, r\left(\mathbf{x}_{l}\right)\right),
\end{aligned}
$$

where the key step is to use the norm equivalence between $\left(\int_{\hat{T}_{2}}\left(\hat{\lambda}_{1}+\hat{\lambda}_{2}\right)^{-1} \hat{v}_{k}^{2} d \hat{r} d \hat{z}\right)^{1 / 2}$ and $\left(\sum_{\hat{\mathbf{x}}_{l} \in \hat{T}_{2}} \hat{v}_{k}^{2}\left(\hat{\mathbf{x}}_{l}\right)\right)^{1 / 2}$. This can be done because the first term is finite for all linear functions that vanish on the $z$-axis.

Hence, based on (52), (55), and (58), we have for any $T_{i} \in \mathcal{T}_{k}$

(60) $\int_{T_{i}}\left(\nabla v_{k} \cdot \nabla v_{k}\right) r d r d z \leq C \sum_{\mathbf{x}_{l} \in T_{i}} v_{k}^{2}\left(\mathbf{x}_{l}\right) \max \left(d_{l, k}, r\left(\mathbf{x}_{l}\right)\right), \quad \forall v_{k} \in \mathbb{V}_{k}^{\beta}$

Summing up (53), (56), and (59), we obtain that for any $v_{k} \in \mathbb{V}_{k}^{1}$ and $T_{i} \in \mathcal{T}_{k}$,

$$
\int_{T_{i}} r^{-1} v_{k}^{2} d r d z \leq C \sum_{\mathbf{x}_{l} \in T_{i}} v_{k}^{2}\left(\mathbf{x}_{l}\right) \max \left(d_{l, k}, r\left(\mathbf{x}_{l}\right)\right) .
$$


Consequently, using (60), (61), and Lemma 4.4, for any $v_{k} \in \mathbb{V}_{k}^{\beta}$, we have

$$
\begin{aligned}
\left(A_{k}^{\beta} v_{k}, v_{k}\right)_{k} & =a_{\beta}\left(v_{k}, v_{k}\right)=\sum_{T_{i} \in \mathcal{T}_{k}} \int_{T_{i}}\left(\nabla v_{k} \cdot \nabla v_{k}+\beta r^{-2} v_{k}^{2}\right) r d r d z \\
& \leq C \sum_{T_{i} \in \mathcal{T}_{k}}\left(\sum_{\mathbf{x}_{l} \in T_{i}} v_{k}^{2}\left(\mathbf{x}_{l}\right) \max \left(d_{l, k}, r\left(\mathbf{x}_{l}\right)\right)\right) \\
& \simeq \sum_{1 \leq i \leq \operatorname{dim}\left(\mathbb{V}_{k}^{\beta}\right)} v_{k}^{2}\left(\mathbf{x}_{i}\right) \max \left(d_{i, k}, r\left(\mathbf{x}_{i}\right)\right) \simeq \operatorname{dim}\left(\mathbb{V}_{k}\right)\left(v_{k}, v_{k}\right)_{k} .
\end{aligned}
$$

Remark 4.6. Based on Lemma 4.5, we can choose the damping factor

$$
\gamma_{k}=\mathcal{O}\left(\operatorname{dim}\left(\mathbb{V}_{\mathrm{k}}^{\beta}\right)^{-1}\right)
$$

for the multigrid V-cycle, such that the spectral radius

$$
0<\rho\left(\gamma_{k} A_{k}^{\beta}\right) \leq 1
$$

4.2. The multigrid analysis. We now concentrate on the convergence analysis of the multigrid V-cycle defined in Algorithm 4.1 for the axisymmetric equation (1).

We first have the smoothing property for the Richardson smoother.

Lemma 4.7. Recall the operator $R_{k}$ from (48). Then,

$$
a_{\beta}\left(\left(I d_{k}-R_{k}\right) R_{k}^{2 l} v_{k}, v_{k}\right) \leq \frac{1}{2 l} a_{\beta}\left(\left(I d_{k}-R_{k}^{2 l}\right) v_{k}, v_{k}\right), \quad \forall v_{k} \in \mathbb{V}_{k}^{\beta} .
$$

Proof. Since $R_{k}=I d_{k}-\gamma_{k} A_{k}^{\beta}$, by (63), for any $0 \leq j \leq i$, we have

$$
\begin{aligned}
a_{\beta}\left(\left(I d_{k}-R_{k}\right) R_{k}^{i} v_{k}, v_{k}\right) & =\gamma_{k}\left(R_{k}^{i} A_{k}^{\beta} v_{k}, A_{k}^{\beta} v_{k}\right)_{k} \\
& \leq \gamma_{k}\left(R_{k}^{j} A_{k}^{\beta} v_{k}, A_{k}^{\beta} v_{k}\right)_{k}=a_{\beta}\left(\left(I d_{k}-R_{k}\right) R_{k}^{j} v_{k}, v_{k}\right) .
\end{aligned}
$$

Then, the lemma is proved by the following telescopic cancellation

$$
\begin{aligned}
(2 l) a_{\beta}\left(\left(I d_{k}-R_{k}\right) R_{k}^{2 l} v_{k}, v_{k}\right) & \leq \sum_{j=0}^{2 l-1} a_{\beta}\left(\left(I d_{k}-R_{k}\right) R_{k}^{j} v_{k}, v_{k}\right) \\
& \leq a_{\beta}\left(\left(I d_{k}-R_{k}^{2 l}\right) v_{k}, v_{k}\right) .
\end{aligned}
$$

Lemma 4.8. The error-propagation operator $E_{k}$ in $(47)$ is symmetric positive semi-definite with respect to $a_{\beta}(\cdot, \cdot)$ for $k \geq 0$.

Proof. We prove it by induction. For $k=0$, the lemma holds. Assume the statement holds for $k-1, k \geq 1$. We first show the symmetry of $E_{k}$. By (47), (48), (49), and the assumption for $E_{k-1}$, we have

$$
\begin{aligned}
& a_{\beta}\left(E_{k} v_{k}, w_{k}\right)=a_{\beta}\left(R_{k}^{l}\left(I d_{k}-P_{k}^{k-1}+E_{k-1} P_{k}^{k-1}\right) R_{k}^{l} v_{k}, w_{k}\right) \\
& =a_{\beta}\left(R_{k}^{l} v_{k},\left(I d_{k}-P_{k}^{k-1}\right) R_{k}^{l} w_{k}\right)+a_{\beta}\left(E_{k-1} P_{k}^{k-1} R_{k}^{l} v_{k}, R_{k}^{l} w_{k}\right) \\
& =a_{\beta}\left(R_{k}^{l} v_{k},\left(I d_{k}-P_{k}^{k-1}\right) R_{k}^{l} w_{k}\right)+a_{\beta}\left(R_{k}^{l} v_{k}, E_{k-1} P_{k-1} R_{k}^{l} w_{k}\right) \\
& =a_{\beta}\left(v_{k}, R_{k}^{l}\left(I d_{k}-P_{k}^{k-1}+E_{k-1} P_{k}^{k-1}\right) R_{k}^{l} w_{k}\right)=a_{\beta}\left(v_{k}, E_{k} w_{k}\right) .
\end{aligned}
$$


We now show that $E_{k}$ is positive and semi-definite. By (47), (49), and the assumption on $E_{k-1}$,

$$
\begin{aligned}
a_{\beta}\left(E_{k} v_{k}, v_{k}\right)= & a_{\beta}\left(R_{k}^{l} v_{k}, R_{k}^{l} v_{k}\right)-a_{\beta}\left(\left(I d_{k}-E_{k-1}\right) P_{k}^{k-1} R_{k}^{l} v_{k}, P_{k}^{k-1} R_{k}^{l} v_{k}\right) \\
= & a_{\beta}\left(\left(I d_{k}-P_{k}^{k-1}\right) R_{k}^{l} v_{k},\left(I d_{k}-P_{k}^{k-1}\right) R_{k}^{l} v_{k}\right) \\
& +a_{\beta}\left(E_{k-1} P_{k}^{k-1} R_{k}^{l} v_{k}, P_{k}^{k-1} R_{k}^{l} v_{k}\right) \geq 0 .
\end{aligned}
$$

In the next two lemmas, we give approximation results needed in the analysis.

Lemma 4.9. Let $\mathcal{E}$ be $H_{+, 0}^{1}(\Omega)$ for $\beta=0$ and be $H_{-, 0}^{1}(\Omega)$ for $\beta=1$. For any $v_{k} \in \mathbb{V}_{k}^{\beta}$ and $k \geq 1$, we have

$$
\left\|\left(I d_{k}-\mathcal{I}_{k-1}^{k} P_{k}^{k-1}\right) v_{k}\right\|_{k} \leq C \operatorname{dim}\left(\mathbb{V}_{k}^{\beta}\right)^{-1 / 2}\left\|\left(I d_{k}-\mathcal{I}_{k-1}^{k} P_{k}^{k-1}\right) v_{k}\right\|_{\mathcal{E}} .
$$

Proof. We now give the proof for the case $\beta=1$. Consider the auxiliary variational problem: Find $\zeta \in H_{-, 0}^{1}(\Omega)$, such that

$$
a_{1}(\zeta, \xi)=\int_{\Omega} \omega_{k}^{2}\left(I d_{k}-\mathcal{I}_{k-1}^{k} P_{k}^{k-1}\right) v_{k} \xi r d r d z, \quad \forall \xi \in H_{-, 0}^{1}(\Omega),
$$

where $a_{1}(\zeta, \xi)=\left(\mathcal{L}_{1} \zeta, \xi\right)_{L_{1}^{2}(\Omega)}$ is the bilinear form in (11) and $\omega_{k}$ is the function defined in (44). Let $\zeta_{k-1} \in \mathbb{V}_{k-1}^{\beta}$ be the finite element solution of (64) on the $k-1$ st level mesh. Recall the layer $L_{j}$ of the triangulation $\mathcal{T}_{k}$ in (43). Then, since the regularity index $0<a_{1}<1$, by Theorem 3.5, (42), (44), and (64), we have

$$
\begin{aligned}
& \left\|\zeta-\mathcal{I}_{k-1}^{k} \zeta_{k-1}\right\|_{H_{-}^{1}(\Omega)}^{2}=\left\|\zeta-\zeta_{k-1}\right\|_{H_{-}^{1}(\Omega)}^{2} \leq C \operatorname{dim}\left(\mathbb{V}_{k-1}^{1}\right)^{-1}\left\|\mathcal{L}_{1} \zeta\right\|_{\mathcal{K}_{a_{1}-1,-}^{0}(\Omega)}^{2} \\
& \simeq \operatorname{dim}\left(\mathbb{V}_{k}^{1}\right)^{-1}\left\|\mathcal{L}_{1} \zeta\right\|_{\mathcal{K}_{a_{1}-1,-}^{0}(\Omega)}^{2}=\operatorname{dim}\left(\mathbb{V}_{k}^{1}\right)^{-1} \sum_{j=0}^{k}\left\|\vartheta^{1-a_{1}} \mathcal{L}_{1} \zeta\right\|_{L_{1}^{2}\left(L_{j}\right)}^{2} \\
& \leq C \operatorname{dim}\left(\mathbb{V}_{k}^{1}\right)^{-1} \sum_{j=0}^{k}\left\|\kappa_{1}^{\left(1-a_{1}\right) j} \mathcal{L}_{1} \zeta\right\|_{L_{1}^{2}\left(L_{j}\right)}^{2}=C \operatorname{dim}\left(\mathbb{V}_{k}^{1}\right)^{-1} \sum_{j=0}^{k}\left\|\kappa_{1}^{j} 2^{j} \mathcal{L}_{1} \zeta\right\|_{L_{1}^{2}\left(L_{j}\right)}^{2} \\
(65) \quad & C \operatorname{dim}\left(\mathbb{V}_{k}^{1}\right)^{-1}\left\|\left(I d_{k}-\mathcal{I}_{k-1}^{k} P_{k}^{k-1}\right) v_{k}\right\|_{k}^{2} .
\end{aligned}
$$

Then, by (50), (65), and Lemma 4.4, we have

$$
\begin{aligned}
& \left\|\left(I d_{k}-\mathcal{I}_{k-1}^{k} P_{k}^{k-1}\right) v_{k}\right\|_{k}^{2}=a_{1}\left(\zeta,\left(I d_{k}-\mathcal{I}_{k-1}^{k} P_{k}^{k-1}\right) v_{k}\right) \\
& =a_{1}\left(\zeta-\mathcal{I}_{k-1}^{k} \zeta_{k-1},\left(I d_{k}-\mathcal{I}_{k-1}^{k} P_{k}^{k-1}\right) v_{k}\right) \\
& \leq\left\|\zeta-\mathcal{I}_{k-1}^{k} \zeta_{k-1}\right\|_{H_{-}^{1}(\Omega)}\left\|\left(I d_{k}-\mathcal{I}_{k-1}^{k} P_{k}^{k-1}\right) v_{k}\right\|_{H_{-}^{1}(\Omega)} \\
& \leq C \operatorname{dim}\left(\mathbb{V}_{k}^{1}\right)^{-1 / 2}\left\|\left(I d_{k}-\mathcal{I}_{k-1}^{k} P_{k}^{k-1}\right) v_{k}\right\|_{k}\left\|\left(I d_{k}-\mathcal{I}_{k-1}^{k} P_{k}^{k-1}\right) v_{k}\right\|_{H_{-}^{1}(\Omega)} .
\end{aligned}
$$

Thus, we obtain for $\beta=1$,

$$
\left\|\left(I d_{k}-\mathcal{I}_{k-1}^{k} P_{k}^{k-1}\right) v_{k}\right\|_{k} \leq C \operatorname{dim}\left(\mathbb{V}_{k}^{1}\right)^{-1 / 2}\left\|\left(I d_{k}-\mathcal{I}_{k-1}^{k} P_{k}^{k-1}\right) v_{k}\right\|_{H_{-}^{1}(\Omega)} .
$$

The proof for $\beta=0$ follows similarly.

Lemma 4.10. Recall the space $\mathcal{E}$ from Lemma 4.9. For any $v_{k} \in \mathbb{V}_{k}^{\beta}$ and $k \geq 1$,

$$
\left\|\left(I d_{k}-\mathcal{I}_{k-1}^{k} P_{k}^{k-1}\right) v_{k}\right\|_{\mathcal{E}} \leq C \operatorname{dim}\left(\mathbb{V}_{k}^{\beta}\right)^{-1 / 2}\left\|A_{k}^{\beta} v_{k}\right\|_{k} .
$$


Proof. Using the Cauchy-Schwarz inequality, (50), and Lemma 4.9, we have

$$
\begin{array}{r}
\left\|\left(I d_{k}-\mathcal{I}_{k-1}^{k} P_{k}^{k-1}\right) v_{k}\right\|_{\mathcal{E}}^{2}=a_{\beta}\left(\left(I d_{k}-\mathcal{I}_{k-1}^{k} P_{k}^{k-1}\right) v_{k},\left(I d_{k}-\mathcal{I}_{k-1}^{k} P_{k}^{k-1}\right) v_{k}\right) \\
=a_{\beta}\left(\left(I d_{k}-\mathcal{I}_{k-1}^{k} P_{k}^{k-1}\right) v_{k}, v_{k}\right)=\left(\left(I d_{k}-\mathcal{I}_{k-1}^{k} P_{k}^{k-1}\right) v_{k}, A_{k}^{\beta} v_{k}\right)_{k} \\
\leq\left\|\left(I d_{k}-\mathcal{I}_{k-1}^{k} P_{k}^{k-1}\right) v_{k}\right\|_{k}\left\|A_{k}^{\beta} v_{k}\right\|_{k} \\
\leq C \operatorname{dim}\left(\mathbb{V}_{k}^{\beta}\right)^{-1 / 2}\left\|\left(I d_{k}-\mathcal{I}_{k-1}^{k} P_{k}^{k-1}\right) v_{k}\right\|_{\mathcal{E}}\left\|A_{k}^{\beta} v_{k}\right\|_{k},
\end{array}
$$

which completes the proof.

Then, we have the estimate for the error-propagation operator $E_{k}$ in (47).

Theorem 4.11. Recall the number of smoothing steps $l$ in Algorithm 4.1. Then,

$$
a_{\beta}\left(E_{k} v_{k}, v_{k}\right) \leq \frac{C}{l+C} a_{\beta}\left(v_{k} . v_{k}\right), \quad \forall v_{k} \in \mathbb{V}_{k}^{\beta},
$$

where $C>0$ is independent of $k$.

Proof. Recall the space $\mathcal{E}$ defined in Lemma 4.9. By Lemma 4.10, (62), and Lemma 4.7 , we first have

$$
\begin{array}{r}
a_{\beta}\left(\left(I d_{k}-P_{k}^{k-1}\right) R_{k}^{l} v_{k},\left(I d_{k}-P_{k}^{k-1}\right) R_{k}^{l} v_{k}\right)=\left\|\left(\left(I d_{k}-P_{k}^{k-1}\right) R_{k}^{l} v_{k}\right)\right\|_{\mathcal{E}}^{2} \\
\leq C \operatorname{dim}\left(\mathbb{V}_{k}^{\beta}\right)^{-1} a_{\beta}\left(A_{k}^{\beta} R_{k}^{l} v_{k}, R_{k}^{l} v_{k}\right)=C \operatorname{dim}\left(\mathbb{V}_{k}^{\beta}\right)^{-1} \gamma_{k}^{-1} a_{\beta}\left(\left(I d_{k}-R_{k}\right) R_{k}^{l} v_{k}, R_{k}^{l} v_{k}\right) \\
\quad \leq C a_{\beta}\left(\left(I d_{k}-R_{k}\right) R_{k}^{2 l} v_{k}, v_{k}\right) \leq \frac{C}{l} a_{\beta}\left(\left(I d_{k}-R_{k}^{2 l}\right) v_{k}, v_{k}\right) .
\end{array}
$$

We now give the proof by induction. For $k=0,(66)$ holds since $E_{0}=0$. Assume (66) holds for $k-1, k \geq 1$. Let $c=C(l+C)^{-1}$ where $C$ is the constant in (67). Note that $c=(1-c) C l^{-1}$. Then, by (47), (49), the assumption on $E_{k-1}$, and (67),

$$
\begin{array}{r}
a_{\beta}\left(E_{k} v_{k}, v_{k}\right)=a_{\beta}\left(\left(I d_{k}-P_{k}^{k-1}\right) R_{k}^{l} v_{k},\left(I d_{k}-P_{k}^{k-1}\right) R_{k}^{l} v_{k}\right) \\
+a_{\beta}\left(E_{k-1} P_{k}^{k-1} R_{k}^{l} v_{k}, P_{k}^{k-1} R_{k}^{l} v_{k}\right) \\
\leq a_{\beta}\left(\left(I d_{k}-P_{k}^{k-1}\right) R_{k}^{l} v_{k},\left(I d_{k}-P_{k}^{k-1}\right) R_{k}^{l} v_{k}\right)+c a_{\beta}\left(P_{k}^{k-1} R^{l} v_{k}, P_{k}^{k-1} R^{l} v_{k}\right) \\
=(1-c) a_{\beta}\left(\left(I d_{k}-P_{k}^{k-1}\right) R_{k}^{l} v_{k},\left(I_{k}-P_{k}^{k-1}\right) R_{k}^{l} v_{k}\right)+c a_{\beta}\left(R^{l} v_{k}, R^{l} v_{k}\right) \\
\leq(1-c) C l^{-1} a_{\beta}\left(\left(I d_{k}-R_{k}^{2 l}\right) v_{k}, v_{k}\right)+c a_{\beta}\left(R^{l} v_{k}, R^{l} v_{k}\right) \\
=c a_{\beta}\left(\left(I d_{k}-R_{k}^{2 l}\right) v_{k}, v_{k}\right)+c a_{\beta}\left(R^{l} v_{k}, R^{l} v_{k}\right)=c a_{\beta}\left(v_{k}, v_{k}\right),
\end{array}
$$

which completes the proof.

Hence, the multigrid $\mathrm{V}$-cycle algorithm is a contraction with contraction number strictly less than 1 , independent of the mesh level $k$.

Theorem 4.12. With the choice of the damping factor $\gamma_{k}$ in (63), the multigrid $V$-cycle scheme defined in Algorithm 4.1 solving the axisymmetric equation (30) converges uniformly,

$$
\left\|E_{k} v_{k}\right\|_{\mathcal{E}} \leq \frac{C}{l+C}\left\|v_{k}\right\|_{\mathcal{E}}, \quad \forall v_{k} \in \mathbb{V}_{k}^{\beta}
$$

where $C$ is independent of the mesh level $k$ and $\mathcal{E}$ is the space in Lemma 4.9.

Proof. By Lemma 4.8, let $0 \leq \alpha_{1} \leq \cdots \leq \alpha_{n_{k}}$ be the eigenvalues of $E_{k}$ and $\nu_{1}, \cdots, \nu_{n_{k}}$ be the corresponding orthonormal eigenvectors with respect to $a_{\beta}(\cdot, \cdot)$. Then, for any $v \in \mathbb{V}_{k}^{\beta}$, we write $v_{k}=\sum_{1 \leq i \leq n_{k}} \omega_{i} \nu_{i}$. Therefore, $a_{\beta}\left(E_{k} v_{k}, v_{k}\right)=$ 
$\sum_{1 \leq i \leq n_{k}} \alpha_{i} \omega_{i}^{2}$. The estimate in (66) indicates that all the eigenvalues $\alpha_{i} \leq C(l+$ $C)^{-1}$. Then, the proof is completed by

$$
\begin{gathered}
\left\|E_{k} v_{k}\right\|_{\mathcal{E}}^{2}=a_{\beta}\left(E_{k} v_{k}, E_{k} v_{k}\right)=\sum_{1 \leq i \leq n_{k}} \alpha_{i}^{2} \omega_{i}^{2} \\
\leq\left(\frac{C}{l+C}\right)^{2} \sum_{1 \leq i \leq n_{k}} \omega_{i}^{2} \leq\left(\frac{C}{l+C}\right)^{2}\left\|v_{k}\right\|_{\mathcal{E}}^{2} .
\end{gathered}
$$

Remark 4.13. The convergence result (Theorem 4.12) is the generalization of the Braess-Hackbusch estimate for self-adjoint second order elliptic equations with the full regularity assumption [7], for axisymmetric equations with singular solutions. It clearly holds when the solution is in $H_{1}^{2}$ and quasi-uniform meshes are used. In particular, the $\mathrm{V}$-cycle algorithm converges with one pre-smoothing and postsmoothing step. The smoothing property (Lemma 4.7) and approximation properties (Lemmas 4.9 and 4.10) are essential. When the solution is singular and only quasi-uniform meshes are used, with the partial-regularity result [6], it is possible to obtain multigrid analysis following the methods in $[9,10,8,28]$. Multigrid algorithms with smoothers other than the Richardson smoother will be investigated in the future.

\section{Numerical Illustrations}

We implemented two sets of numerical tests for the multigrid V-cycle algorithm (Algorithm 4.1) with different values of $\beta$ on different domains. In all these tests, based on the regularity result in Theorem 3.5, suitable graded or quasi-uniform meshes are chosen to achieve the optimal convergence rate in the finite element solution, as indicated in Theorem 3.16. It is shown in all the cases, that the proposed multigrid V-cycle algorithms are contractions with contraction numbers decreasing as the number of smoothing steps $l$ increases, but independent of mesh level $k$, which convincingly verifies Theorem 4.12.

5.1. Numerical tests. The first set of tests is for equation (30) with $\beta=0$ on three domains (Figure 3): the square $\Omega_{1}$, the L-shape domain $\Omega_{2}$, and the polygon with a $170^{\circ}$ interior angle on the $z$-axis $\Omega_{3}$. By the calculation of regularity indices (Remarks 3.2 and 3.4), given $f \in L_{1}^{2}$, the corresponding solutions satisfy $u_{0} \in$ $H_{1}^{2}\left(\Omega_{1}\right), u_{0} \in \mathcal{K}_{a_{0}+1}^{2}\left(\Omega_{2}\right)$ for $a_{0}<\eta_{0}=\frac{2}{3}$, and $u_{0} \in \mathcal{K}_{a_{0}+1}^{2}\left(\Omega_{3}\right)$ for $a_{0}<\eta_{0} \approx$ 0.7. Consequently, the linear finite element approximation obtains the optimal convergence rate on quasi-uniform meshes for $\Omega_{1}$ and on graded meshes toward the vertex $Q$ with $\kappa_{0}<0.354$ for $\Omega_{2}$ and $\kappa_{0}<0.372$ for $\Omega_{3}$. Some examples of optimal meshes are given in Figure 3.

The contraction numbers of the proposed multigrid V-cycle algorithms are listed in Tables 1-3, where $k$ denotes the mesh level and $l$ is the number of pre-smoothing (post-smoothing) steps. It is clear that for fixed $l$, the contraction numbers are independent of the mesh level $k$ for all domains with optimal meshes. Note that the contraction number becomes smaller when we increase the number of smoothing steps. This is also predicted by Theorem 4.12 .

The second set of tests is for equation (30) with $\beta=1$ on two domains (Figure 3 ): the square $\Omega_{1}$ and the L-shape domain $\Omega_{2}$. For $f_{1} \in L_{1}^{2}$, our regularity result implies that the solutions $u_{1} \in H_{1}^{2}\left(\Omega_{1}\right)$ and $u_{1} \in \mathcal{K}_{a_{1}+1}^{2}\left(\Omega_{2}\right)$ for $a_{1}<\eta_{1}=\frac{2}{3}$. 

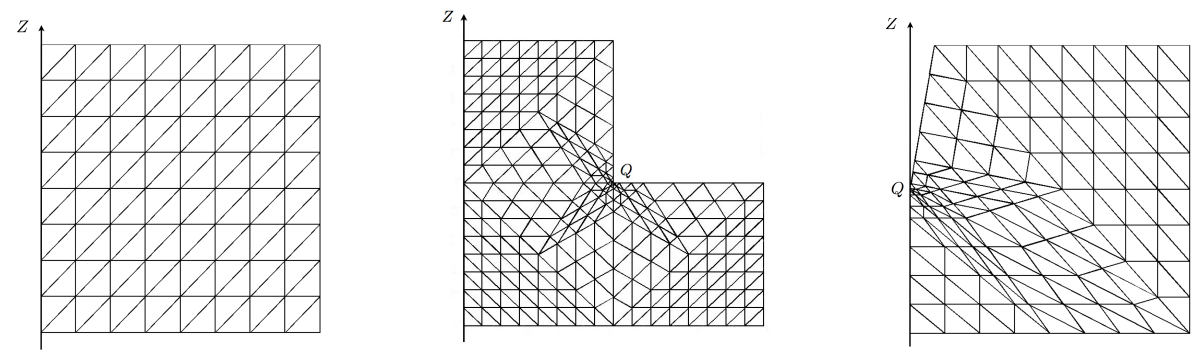

Figure 3. The quasi-uniform mesh for the square domain $\Omega_{1}$ (left); the graded mesh for the L-shape domain $\Omega_{2}, \kappa=0.2$ (center); the graded mesh for the domain with the $170^{\circ}$ interior angle $\Omega_{3}, \kappa=0.2$ (right).

\begin{tabular}{|r|lllllll|}
\hline$(l, l) \backslash k$ & $k=1$ & $k=2$ & $k=3$ & $k=4$ & $k=5$ & $k=6$ & $k=7$ \\
\hline $1-1$ & 0.72 & 0.72 & 0.73 & 0.73 & 0.73 & 0.73 & 0.73 \\
\hline $2-2$ & 0.52 & 0.53 & 0.53 & 0.53 & 0.53 & 0.53 & 0.53 \\
\hline $4-4$ & 0.28 & 0.30 & 0.30 & 0.30 & 0.30 & 0.30 & 0.30 \\
\hline $8-8$ & 0.11 & 0.12 & 0.12 & 0.12 & 0.12 & 0.12 & 0.12 \\
\hline
\end{tabular}

TABLE 1. Contraction numbers of the multigrid V-cycle algorithm for equation (30) $(\beta=0)$ on $\Omega_{1}$ with uniform meshes (Figure 3).

\begin{tabular}{|r|lllllll|}
\hline$(l, l) \backslash k$ & $k=1$ & $k=2$ & $k=3$ & $k=4$ & $k=5$ & $k=6$ & $k=7$ \\
\hline $1-1$ & 0.51 & 0.51 & 0.56 & 0.60 & 0.62 & 0.64 & 0.64 \\
\hline $2-2$ & 0.26 & 0.37 & 0.44 & 0.47 & 0.49 & 0.51 & 0.52 \\
\hline $4-4$ & 0.13 & 0.25 & 0.31 & 0.34 & 0.36 & 0.37 & 0.38 \\
\hline $8-8$ & 0.05 & 0.14 & 0.19 & 0.22 & 0.23 & 0.24 & 0.25 \\
\hline
\end{tabular}

TABLE 2. Contraction numbers of the multigrid V-cycle algorithm for equation $(30)(\beta=0)$ on $\Omega_{2}, \kappa_{0}=0.2$ (Figure 3$)$.

\begin{tabular}{|r|lllllll|}
\hline$(l, l) \backslash k$ & $k=1$ & $k=2$ & $k=3$ & $k=4$ & $k=5$ & $k=6$ & $k=7$ \\
\hline $1-1$ & 0.85 & 0.87 & 0.90 & 0.91 & 0.91 & 0.92 & 0.92 \\
\hline $2-2$ & 0.73 & 0.78 & 0.81 & 0.83 & 0.84 & 0.85 & 0.85 \\
\hline $4-4$ & 0.56 & 0.64 & 0.69 & 0.72 & 0.74 & 0.75 & 0.75 \\
\hline $8-8$ & 0.36 & 0.47 & 0.54 & 0.58 & 0.60 & 0.61 & 0.62 \\
\hline
\end{tabular}

TABLE 3. Contraction numbers of the multigrid V-cycle algorithm for equation $(30)(\beta=0)$ on $\Omega_{3}, \kappa_{0}=0.2$ (Figure 3 ).

In particular, for a polygonal domain, the possible $H_{1}^{2}$-singularity in the solution only appears near vertices away from the $z$-axis. Namely, $u_{1}$ is always in $H_{1}^{2}$ near vertices on the $z$-axis. This is the reason that we do not consider the solution on $\Omega_{3}$ in this set, since it will have the same character as the solution on the square. Then, the optimal meshes for the linear finite element approximations are quasi-uniform on $\Omega_{1}$ and graded with $\kappa_{1}<0.354$ toward the vertex $Q$ on $\Omega_{2}$. 


\begin{tabular}{|r|lllllll|}
\hline$(l, l) \backslash k$ & $k=1$ & $k=2$ & $k=3$ & $k=4$ & $k=5$ & $k=6$ & $k=7$ \\
\hline $1-1$ & 0.32 & 0.39 & 0.43 & 0.44 & 0.44 & 0.44 & 0.44 \\
\hline $2-2$ & 0.14 & 0.22 & 0.26 & 0.27 & 0.28 & 0.28 & 0.28 \\
\hline $4-4$ & 0.06 & 0.11 & 0.14 & 0.15 & 0.16 & 0.16 & 0.17 \\
\hline $8-8$ & 0.03 & 0.05 & 0.07 & 0.08 & 0.09 & 0.09 & 0.09 \\
\hline
\end{tabular}

TABLE 4. Contraction numbers of the multigrid V-cycle algorithm for equation (30) $(\beta=1)$ on $\Omega_{1}$ with uniform meshes (Figure 3).

\begin{tabular}{|r|lllllll|}
\hline$(l, l) \backslash k$ & $k=1$ & $k=2$ & $k=3$ & $k=4$ & $k=5$ & $k=6$ & $k=7$ \\
\hline $1-1$ & 0.34 & 0.49 & 0.56 & 0.60 & 0.62 & 0.63 & 0.64 \\
\hline $2-2$ & 0.22 & 0.36 & 0.43 & 0.47 & 0.49 & 0.51 & 0.51 \\
\hline $4-4$ & 0.11 & 0.23 & 0.30 & 0.33 & 0.35 & 0.37 & 0.38 \\
\hline $8-8$ & 0.03 & 0.12 & 0.18 & 0.21 & 0.23 & 0.24 & 0.25 \\
\hline
\end{tabular}

TABLE 5. Contraction numbers of the multigrid V-cycle algorithm for equation (30) $(\beta=1)$ on $\Omega_{2}, \kappa_{1}=0.2$ (Figure 3).

The contraction numbers of the V-cycle algorithms for $\beta=1$ are listed in Tables 4-5. Similar to the case of $\beta=0$, the algorithms converge uniformly independent of the mesh level $k$. These contraction numbers also well represent the asymptotic rate of decay with respect to the number of smoothing steps, given in Theorem 4.12 .

5.2. Summary. We tested the multigrid algorithm for different values of $\beta$, with both regular and singular solutions. All the numerical tests clearly show the uniform convergence of the multigrid algorithm. The estimates of the regularity in weighted spaces and finite element approximations on graded meshes are the ingredients of our multigrid analysis.

\section{REFERENCES}

[1] F. Assous, P. Ciarlet, Jr., and S. Labrunie. Theoretical tools to solve the axisymmetric Maxwell equations. Math. Methods Appl. Sci., 25(1):49-78, 2002.

[2] C. Băcuţă, V. Nistor, and L. Zikatanov. Improving the rate of convergence of high-order finite elements on polyhedra. I. A priori estimates. Numer. Funct. Anal. Optim., 26(6):613-639, 2005.

[3] C. Băcuţă, V. Nistor, and L. T. Zikatanov. Improving the rate of convergence of 'high order finite elements' on polygons and domains with cusps. Numer. Math., 100(2):165-184, 2005.

[4] C. Bacuta, V. Nistor, and L.T. Zikatanov. Improving the rate of convergence of high-order finite elements on polyhedra. II. Mesh refinements and interpolation. Numer. Funct. Anal. Optim., 28(7-8):775-824, 2007.

[5] Z. Belhachmi, C. Bernardi, and S. Deparis. Weighted Clément operator and application to the finite element discretization of the axisymmetric Stokes problem. Numer. Math., 105(2):217$247,2006$.

[6] C. Bernardi, M. Dauge, and Y. Maday. Spectral methods for axisymmetric domains. Series in Applied Mathematics. Gauthier-Villars, North Holland, Paris, Amsterdam, 1999.

[7] D. Braess and W. Hackbusch. A new convergence proof for the multigrid method including the $V$-cycle. SIAM J. Numer. Anal., 20(5):967-975, 1983.

[8] J.H. Bramble and J.E. Pasciak. New estimates for multilevel algorithms including the $V$-cycle. Math. Comp., 60(202):447-471, 1993.

[9] J.H. Bramble, J.E. Pasciak, J. Wang, and J. Xu. Convergence estimates for multigrid algorithms without regularity assumptions. Math. Comp., 57(195):23-45, 1991. 
[10] S.C. Brenner. Convergence of the multigrid $V$-cycle algorithm for second-order boundary value problems without full elliptic regularity. Math. Comp., 71(238):507-525 (electronic), 2002.

[11] S.C. Brenner and L.R. Scott. The mathematical theory of finite element methods, volume 15 of Texts in Applied Mathematics. Springer-Verlag, New York, second edition, 2002.

[12] D.M. Copeland, J. Gopalakrishnan, and J.E. Pasciak. A mixed method for axisymmetric div-curl systems. Math. Comp., 77(264):1941-1965, 2008.

[13] M. Dauge. Elliptic Boundary Value Problems on Corner Domains, volume 1341 of Lecture Notes in Mathematics. Springer-Verlag, Berlin, 1988.

[14] L. Evans. Partial differential equations, volume 19 of Graduate Studies in Mathematics. AMS, Rhode Island, 1998.

[15] J. Gopalakrishnan and J.E. Pasciak. The convergence of V-cycle multigrid algorithms for axisymmetric Laplace and Maxwell equations. Math. Comp., 75(256):1697-1719 (electronic), 2006.

[16] P. Grisvard. Singularities in boundary value problems, volume 22 of Research Notes in Applied Mathematics. Springer-Verlag, New York, 1992.

[17] B. Heinrich. The Fourier-finite-element method for Poisson's equation in axisymmetric domains with edges. SIAM J. Numer. Anal., 33(5):1885-1911, 1996.

[18] V.A. Kondrat'ev. Boundary value problems for elliptic equations in domains with conical or angular points. Trudy Moskov. Mat. Ob̌̌č., 16:209-292, 1967.

[19] V. Kozlov, V. Maz'ya, and J. Rossmann. Elliptic boundary value problems in domains with point singularities, volume 52 of Mathematical Surveys and Monographs. American Mathematical Society, Providence, RI, 1997.

[20] V. Kozlov, V. Maz'ya, and J. Rossmann. Spectral problems associated with corner singularities of solutions to elliptic equations, volume 85 of Mathematical Surveys and Monographs. American Mathematical Society, Providence, RI, 2001.

[21] Y.-J. Lee and H. Li. On stability, accuracy, and fast solvers for finite element approximations of the axisymmetric stokes problem by hood-taylor elements. SIAM J. Numer. Anal., 49(2):668-691, 2011.

[22] Y.-J. Lee and H. Li. Axisymmetric Stokes equations in polygonal domains: Regularity and finite element approximations. Comput. Math. Appl., 64(11):3500-3521, 2012.

[23] H. Li. Finite element analysis for the axisymmetric Laplace operator on polygonal domains. J. Comput. Appl. Math., 235(17):5155-5176, 2011.

[24] H. Li, A. Mazzucato, and V. Nistor. Analysis of the finite element method for transmission/mixed boundary value problems on general polygonal domains. Electron. Trans. Numer. Anal., 37:41-69, 2010.

[25] H. Li and V. Nistor. Analysis of a modified Schrödinger operator in 2D: regularity, index, and FEM. J. Comput. Appl. Math., 224(1):320-338, 2009.

[26] B. Mercier and G. Raugel. Résolution d'un problème aux limites dans un ouvert axisymétrique par éléments finis en $r, z$ et séries de Fourier en $\theta$. RAIRO Anal. Numér., 16(4):405-461, 1982.

[27] B. Nkemzi. The Poisson equation in axisymmetric domains with conical points. J. Comput. Appl. Math., 174(2):399-421, 2005.

[28] J. Xu and L. Zikatanov. The method of alternating projections and the method of subspace corrections in Hilbert space. J. Amer. Math. Soc., 15(3):573-597 (electronic), 2002.

Hengguang Li, Department of Mathematics, Wayne State University, Detroit, Mi 48202, USA

E-mail address: hli@math.wayne.edu 